\title{
Nonlocal strong forms of thin plate, gradient elasticity, magneto-electro-elasticity and phase-field fracture by nonlocal operator method
}

\author{
Huilong Ren ${ }^{3} \cdot$ Xiaoying Zhuang $^{4,5} \cdot$ Erkan Oterkus $^{6} \cdot$ Hehua Zhu $^{5} \cdot$ Timon Rabczuk $^{1,2}$
}

Received: 21 May 2021 / Accepted: 12 August 2021 / Published online: 15 September 2021

(c) The Author(s) 2021

\begin{abstract}
The derivation of nonlocal strong forms for many physical problems remains cumbersome in traditional methods. In this paper, we apply the variational principle/weighted residual method based on nonlocal operator method for the derivation of nonlocal forms for elasticity, thin plate, gradient elasticity, electro-magneto-elasticity and phase-field fracture method. The nonlocal governing equations are expressed as an integral form on support and dual-support. The first example shows that the nonlocal elasticity has the same form as dual-horizon non-ordinary state-based peridynamics. The derivation is simple and general and it can convert efficiently many local physical models into their corresponding nonlocal forms. In addition, a criterion based on the instability of the nonlocal gradient is proposed for the fracture modelling in linear elasticity. Several numerical examples are presented to validate nonlocal elasticity and the nonlocal thin plate.
\end{abstract}

Keywords Energy form $\cdot$ Weak form $\cdot$ Variational principle $\cdot$ Peridynamics $\cdot$ Dual-support $\cdot$ Fracture $\cdot$ Explicit time integration

\section{Introduction}

Classical continuum mechanics has achieved great success in describing the macro-scale properties of solid material based on the continuous medium hypothesis that the material is a continuous mass rather than as discrete particles. The assumption indicates that the substance of the object

Timon Rabczuk

timon.rabczuk@tdtu.edu.vn

1 Division of Computational Mechanics, Ton Duc Thang University, Ho Chi Minh City, Viet Nam

2 Faculty of Civil Engineering, Ton Duc Thang University, Ho Chi Minh City, Viet Nam

3 Institute of Structural Mechanics, Bauhaus-University Weimar, 99423 Weimar, Germany

4 Institute of Continuum Mechanics, Leibniz University Hannover, Hannover, Germany

5 State Key Laboratory of Disaster Reduction in Civil Engineering, College of Civil Engineering, Tongji University, Shanghai 200092, China

6 Department of Naval Architecture, Ocean and Marine Engineering, PeriDynamics Research Centre, University of Strathclyde, 100 Montrose Street, Glasgow G4 OLZ, UK completely fills the space it occupies, without considering the inherent micro-structure of the material. Such a continuous medium hypothesis is not always valid in solid medium. Over the years, researchers found that many phenomena, such as size effect [1], length scale effect [2], skin/edge effect [3], can not be well predicted by traditional continuum mechanics. These phenomena may be attributed to the nonlocal effect in the solid. In contrast with local theory whose mathematical language is partial differential derivatives defined at an infinitesimal point, nonlocal theory is formulated as integral form in a domain.

Classical continuum mechanics is regarded as a local theory. For solid mediums of multiple materials with a material interface or discontinuity such as fracture, the partial differential operator is no longer well defined. Around the fracture front tip, the stress singularity happens for local theory. To model fracture and its evolution, various local theories have been proposed, for example, finite element method (FEM) [4], extended finite element method [5], phase-field fracture method [6-8], cracking particle method $[9,10]$, extended finite element method [11], numerical manifold method [12], extended isogeometric analysis (XIGA) for three-dimensional crack [13], meshfree methods [14-16]. Another approach for fracture modeling is the nonlocal 
method. Compared with classical continuum mechanics without length scale, nonlocal theory takes into account the length scale explicitly and it is less sensitive to the inhomogeneity/discontinuity encountered in the materials due to its integral form.

Two general theories to account for the length scale of solid material, are the gradient elasticity $[1,17-20]$ and the nonlocal elasticity [21-24]. The gradient elasticity theory can be traced back to Cosserat theory in 1909 [25]. It incorporates the length scale and higher order derivative of the displacement field. A variety of gradient elasticity theories have been proposed such as Mindlin solid theory [2, 17], couple stress theory $[1,26]$, modified couple stress $[18$, 27] and second-grade materials [19]. In nonlocal elasticity, the stress tensor is based on the integral of the "local" stress field in a domain, in contrast with the local elasticity defining the stress based on the strain field at a point. Under certain circumstances, the nonlocal elasticity can be transformed into gradient elasticity [23, 28].

Among various nonlocal elasticity theories, peridynamics (PD) $[29,30]$ has attracted the attention of the researchers in the fracture mechanics field. PD is based on the integral form well defined in domain with/without discontinuity. This salient feature enables PD a versatile method for fracture modeling [31-34]. The origin of PD is the bondbased PD (BB-PD) with the Poisson ratio restriction. BB-PD can model 2D elasticity with Poisson ratio of $1 / 3$ and $3 D$ elasticity with Poisson ratio of $1 / 4$. Many efforts have been dedicated to overcome this restriction, for example, PD with shear deformation [35], bond-rotation effect by [36], PD with micropolar deformation [37]. The further development of PD is the state-based PD [30, 38]. Several treatments are developed to overcome the instability issue in non-ordinary state-based PD (NOSBPD), including, bond-associated higher-order stabilized model [39], higher-order approximation [40], stabilized non-ordinary state-based PD [41, 42], sub-horizon scheme [43] and stress point method [44].

In the spirit of nonlocality, PD has been extended in many directions, for example, dual-horizon PD [45, 46], peridynamic plate/shell theory [47-50], mixed peridynamic Petrov-Galerkin method for compressible and incompressible hyperelastic material [51, 52], phase-field-based peridynamic damage model for composite structures [53], wave dispersion analysis of PD [54], damage mechanism in PD [55], coupling scheme for state-based PD and FEM [56, 57], higher-order peridynamic material models for elasticity [58] and Peridynamic differential operator (PDDO) [59-61] for solving partial differential equations, to name a few. PDDO has greatly extended the power of peridynamics and was applied to numerous challenging problems including fluid flow coupled with heat transfer [62] and fracture evolution in batteries [63] among others.
Dual-horizon PD overcomes the restriction of constant horizon in PD, without introducing side effects for variable horizons. Dual-horizon peridynamic formulation can be derived from the Euler-Lagrange equations [64]. Based on the concept in nonlocal theory, we developed the Nonlocal Operator Method (NOM) as the generalization of dual-horizon PD. NOM uses the nonlocal operators of integral form to replace the local partial differential operators of different orders. There are three versions of NOM, first-order particlebased NOM [65, 66], higher-order particle-based NOM [67] and higher-order NOM based on numerical integration [68]. The particle-based version can be viewed as a special case of NOM with numerical integration when nodal integration is employed. The nonlocal operators can be viewed as an alternative to the partial derivatives of shape functions in FEM. Combined with a variational principle or weighted residual method, NOM obtains the residual vector and tangent stiffness matrix in the same way as in FEM. NOM has been applied to the solutions of the Poisson equation in high dimensional space, von-Karman thin plate equations, fracture problems based on phase field [67], waveguide problem in electromagnetic field [66], gradient solid problem [68] and Cahn-Hilliard equation [69].

Although much progress in nonlocal methods has been achieved in the above mentioned literatures, the derivations for many physical problems remain cumbersome and complicated, see for example [48, 58, 70, 71]. In local theory, the local differential operator is a fundamental element for describing physical problems. In analogy, the nonlocal operators would be very beneficial for developing nonlocal theoretical models. The power of NOM in deriving nonlocal models remains largely unexplored. In addition, NOM based on implicit algorithms is relatively complicated in implementation and in this paper, we explore the explicit algorithm in solving the nonlocal models. Furthermore, we propose an instability criterion of the nonlocal gradient operator for the purpose of fracture modeling.

The remaining of the paper is outlined as follows. In Sect. 2, the second-order NOM in 2D/3D is formulated in detail. In Sect. 3, we apply the NOM scheme combined with variational principle/weighted residual method to derive the nonlocal governing equations for elasticity, thin plate, gradient elasticity, electro-magneto-elasticity and phase-field fracture model. The correspondence between local form and nonlocal form for higher-order problems is discussed. In Sect. 4, an instability criterion of nonlocal gradient is presented in the fracture modeling of linear elastic solid. The implementation of nonlocal solid and nonlocal thin plate is discussed in Sect. 5. Several numerical examples for solid and thin plate are used to demonstrate the accuracy and efficiency of the current method in Sect. 6. Last but not the least, some concluding remarks are presented. 


\section{Second-order nonlocal operator method}

NOM uses the integral form to replace the partial differential derivatives of different orders. Although NOM can solve higher order linear/nonlinear problems in 2D/3D, we restrict our discussion in second-order NOM, which is sufficient for the nonlocal derivation of the physical problems to be studied in Sect. 3.

\subsection{Support and dual-support}

Consider a domain as shown in Fig. 1a, let $\boldsymbol{x}_{i}$ be spatial coordinates in the domain $\Omega ; \boldsymbol{r}_{i j}:=\boldsymbol{x}_{j}-\boldsymbol{x}_{i}$ is a spatial vector starting from $x_{i}$ to $x_{j} ; v_{i}:=v\left(x_{i}, t\right)$ and $v_{j}:=v\left(x_{j}, t\right)$ are the field values for $x_{i}$ and $x_{j}$, respectively; $v_{i j}:=v_{j}-v_{i}$ is the relative field vector for spatial vector $\boldsymbol{r}_{i j}$.

Support $\mathcal{S}_{i}$ is the neighbourhood of point $\boldsymbol{x}_{i}$. A point $\boldsymbol{x}_{j}$ in support $\mathcal{S}_{i}$ forms the spatial vector $\boldsymbol{r}_{i j}\left(=\boldsymbol{x}_{j}-\boldsymbol{x}_{i}\right)$. The support in NOM can be a spherical domain, a cube, semi-spherical domain and so on.

Dual-support is defined as a union of points whose supports include $\boldsymbol{x}_{i}$, denoted by

$\mathcal{S}_{i}^{\prime}=\left\{x_{j} \mid x_{i} \in \mathcal{S}_{j}\right\}$.

Point $\boldsymbol{x}_{j}$ forms the dual-vector $\boldsymbol{r}_{j i}\left(=\boldsymbol{x}_{i}-\boldsymbol{x}_{j}=-\boldsymbol{r}_{i j}\right)$ in $\mathcal{S}_{i}^{\prime}$. On the other hand, $\boldsymbol{r}_{j i}$ is the spatial vector formed in $\mathcal{S}_{j}$. It is worth mentioning that the size of the support of each point can be different. When the support sizes for all material points are the same, the dual-support is equal to the support. On the other hand, if the size of support varies for each point, the shape of dual-support can be quite irregular, even discontinuous for two adjacent points. One example to illustrate the support and dual-support is shown in Fig. 1b.

\subsection{Dual property of dual-support}

For point $j \in \mathcal{S}_{i}$, let $f_{i j}$ be a physical quantity, work conjugate to field difference $\left(u_{j}-u_{i}\right)$, the dual property of dualsupport is

$\int_{\Omega} \int_{\mathcal{S}_{i}} f_{i j}\left(u_{j}-u_{i}\right) \mathrm{d} V_{j} \mathrm{~d} V_{i}=\int_{\Omega}\left(\int_{\mathcal{S}_{i}^{\prime}} f_{j i} \mathrm{~d} V_{j}-\int_{\mathcal{S}_{i}} f_{i j} \mathrm{~d} V_{j}\right) u_{i} \mathrm{~d} V_{i}$.

Proof: Let the domain $\Omega$ be divided into $N$ non-overlapping particles, so that $\Omega=\sum_{i=1}^{N} \Delta V_{i}$, where $\Delta V_{i}$ is the volume assigned to particle $i$. Herein, $N$ can be arbitrarily large so that the $\Delta V_{i}$ is infinitesimal and the double summations of discrete form converge to the double integrals in continuous form.

$$
\begin{aligned}
\int_{\Omega} & \int_{\mathcal{S}_{i}} f_{i j}\left(u_{j}-u_{i}\right) \mathrm{d} V_{j} \mathrm{~d} V_{i} \\
& \approx \sum_{\Delta V_{i} \in \Omega} \sum_{\Delta V_{j} \in \mathcal{S}_{i}} f_{i j}\left(u_{j}-u_{i}\right) \Delta V_{j} \Delta V_{i} \\
& =\sum_{\Delta V_{i} \in \Omega} \sum_{\Delta V_{j} \in \mathcal{S}_{i}} f_{i j} u_{j} \Delta V_{j} \Delta V_{i}-\sum_{\Delta V_{i} \in \Omega} \sum_{\Delta V_{j} \in \mathcal{S}_{i}} f_{i j} u_{i} \Delta V_{j} \Delta V_{i} \\
& =\sum_{\Delta V_{i} \in \Omega} \sum_{\Delta V_{j} \in \mathcal{S}_{i}^{\prime}} f_{j i} u_{i} \Delta V_{j} \Delta V_{i}-\sum_{\Delta V_{i} \in \Omega} \sum_{\Delta V_{j} \in \mathcal{S}_{i}} f_{i j} u_{i} \Delta V_{j} \Delta V_{i} \\
& \approx \int_{\Omega}\left(\int_{\mathcal{S}_{i}^{\prime}} f_{j i} \mathrm{~d} V_{j}-\int_{\mathcal{S}_{i}} f_{i j} \mathrm{~d} V_{j}\right) u_{i} \mathrm{~d} V_{i}
\end{aligned}
$$

In the third step, the dual-support is considered as follows. The term $f_{i j}$ with $u_{j}$ is the physical quantity from $i$ 's support, but is added to particle $j$; since $j \in \mathcal{S}_{i}, i$ belongs to the dualsupport $\mathcal{S}_{j}^{\prime}$ of $j$; all terms $f_{j i}$ with $u_{i}$ are collected from any material point $j$ whose support contains $i$ and hence form the dual-support of $i$. Therefore, the dual property of the dual-support is proved.
Fig. 1 a Domain and notation. b Schematic diagram for support and dual-support, all shapes above are supports, $\mathcal{S}_{\boldsymbol{x}}=\left\{\boldsymbol{x}_{1}, \boldsymbol{x}_{2}, \boldsymbol{x}_{4}\right\}$, $\mathcal{S}_{x}^{\prime}=\left\{x_{1}, x_{2}, x_{3}\right\}$

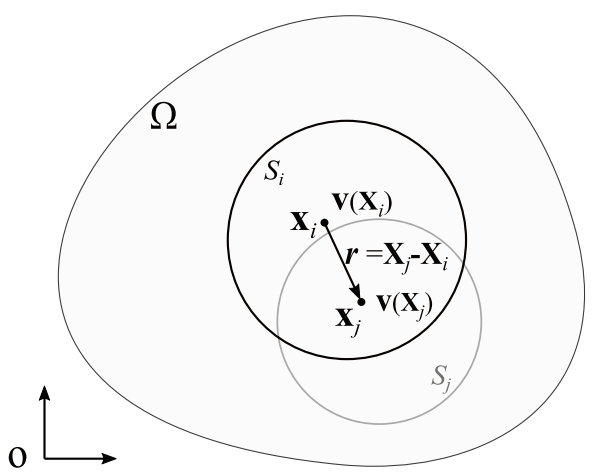

(a)

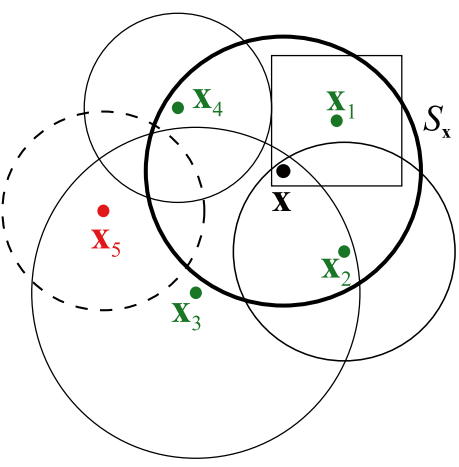

(b) 
When all points have the same size of support domains, i.e. $j \in \mathcal{S}_{i} \leftrightarrow i \in \mathcal{S}_{j}$, we have $\mathcal{S}_{i}=\mathcal{S}_{i}^{\prime}$ for any point $i$ and then the dual property of dual-support by Eq. 2 becomes

$\int_{\Omega} \int_{\mathcal{S}_{i}} f_{i j}\left(u_{j}-u_{i}\right) \mathrm{d} V_{j} \mathrm{~d} V_{i}=\int_{\Omega} \int_{\mathcal{S}_{i}}\left(f_{j i}-f_{i j}\right) u_{i} \mathrm{~d} V_{j} \mathrm{~d} V_{i}$.

Above equation is widely used in the derivation of nonlocal strong form from weak form. Such expression is valid in the continuum form as well as in discrete form. The dual property of dual-support is also proved in the dual-horizon peridynamics [46]. A simple example with $N=4$ to illustrate this property is given in Appendix A.

\subsection{Nonlocal gradient and Hessian operator}

The local gradient operator and Hessian operator for a scalar-valued function $u$ have the forms in 2D

$\nabla u=\left(u_{, x}, u_{, y}\right)^{T}, \quad \nabla^{2} u=\left(\begin{array}{ll}u_{, x x} & u_{, x y} \\ u_{, x y} & u_{, y y}\end{array}\right)$

and in 3D

$\nabla u=\left(u_{, x}, u_{, y}, u_{, z}\right)^{T}, \quad \nabla^{2} u=\left(\begin{array}{lll}u_{, x x} & u_{, x y} & u_{, x z} \\ u_{, x y} & u_{, y y} & u_{, y z} \\ u_{, x z} & u_{, y z} & u_{, z z}\end{array}\right)$

where $u_{, x x}$ denotes the partial derivative of $u$ with respect to $x$ twice.

In the framework of NOM, the partial derivatives can be constructed as follows. The Taylor series expansion of scalar-valued field $u_{j}$ in $2 \mathrm{D}$ can be written as

$u_{j}=u_{i}+\left(u_{i, x}, u_{i, y}, u_{i, x x}, u_{i, x y}, u_{i, y y}\right) \cdot\left(x_{i j}, y_{i j}, x_{i j}^{2} / 2, x_{i j} y_{i j}, y_{i j}^{2} / 2\right)+O\left(\left|\boldsymbol{r}_{i j}\right|^{3}\right)$

where $\boldsymbol{r}_{i j}=\left(x_{i j}, y_{i j}\right)^{T}=\boldsymbol{x}_{j}-\boldsymbol{x}_{i}$ and $O\left(\left|\boldsymbol{r}_{i j}\right|^{3}\right)$ denotes the higher order terms.

Let

$u_{i j}=u_{j}-u_{i}$

$\boldsymbol{p}_{i j}=\left(x_{i j}, y_{i j}, x_{i j}^{2} / 2, x_{i j} y_{i j}, y_{i j}^{2} / 2\right)^{T}$

$\partial u_{i}=\left(u_{i, x}, u_{i, y}, u_{i, x x}, u_{i, x y}, u_{i, y y}\right)^{T}$

The Taylor series expansion of Eq. 7 can be rewritten as

$u_{i j}=\partial u_{i}^{T} \boldsymbol{p}_{i j}$

Tensor product with $\boldsymbol{p}_{i j}^{T}$ on both sides of Eq. 11

$u_{i j} \boldsymbol{p}_{i j}^{T}=\partial u_{i}^{T} \boldsymbol{p}_{i j} \boldsymbol{p}_{i j}^{T}$
Considering the weighted integration in the support $\mathcal{S}_{i}$, we obtain

$\int_{\mathcal{S}_{i}} \omega\left(\boldsymbol{r}_{i j}\right) u_{i j} \boldsymbol{p}_{i j}^{T} \mathrm{~d} V_{j}=\partial u_{i}^{T} \int_{\mathcal{S}_{i}} \omega\left(\boldsymbol{r}_{i j}\right) \boldsymbol{p}_{i j} \boldsymbol{p}_{i j}^{T} \mathrm{~d} V_{j}$

where $\omega\left(\boldsymbol{r}_{i j}\right)$ is the weight function.

Then the nonlocal operators can be obtained as

$\tilde{\partial} u_{i}:=\int_{\mathcal{S}_{i}} \omega\left(\boldsymbol{r}_{i j}\right) \boldsymbol{K}_{i} \cdot \boldsymbol{p}_{i j} u_{i j} \mathrm{~d} V_{j}$

where

$\boldsymbol{K}_{i}=\left(\int_{\mathcal{S}_{i}} \omega\left(\boldsymbol{r}_{i j}\right) \boldsymbol{p}_{i j} \otimes \boldsymbol{p}_{i j}^{T} \mathrm{~d} V_{j}\right)^{-1}$

Here, we use $\tilde{\square}$ to denote the nonlocal form of the local operator $\quad \square$ since the definitions of the local operator and the nonlocal operator are distinct.

The Taylor series expansion of a vector field $\boldsymbol{u}$ can be obtained in the similar manner as

$\boldsymbol{u}_{i j}^{T}=\boldsymbol{p}_{i j}^{T} \cdot \partial \boldsymbol{u}_{i}$

$\omega\left(\boldsymbol{r}_{i j}\right) \boldsymbol{p}_{i j} \otimes \boldsymbol{u}_{i j}^{T}=\omega\left(\boldsymbol{r}_{i j}\right) \boldsymbol{p}_{i j} \otimes \boldsymbol{p}_{i j}^{T} \cdot \partial \boldsymbol{u}_{i}$

$\int_{\mathcal{S}_{i}} \omega\left(\boldsymbol{r}_{i j}\right) \boldsymbol{p}_{i j} \otimes \boldsymbol{u}_{i j}^{T} \mathrm{~d} V_{j}=\int_{\mathcal{S}_{i}} \omega\left(\boldsymbol{r}_{i j}\right) \boldsymbol{p}_{i j} \otimes \boldsymbol{p}_{i j}^{T} \cdot \partial \boldsymbol{u}_{i} \mathrm{~d} V_{j}$

That is

$\tilde{\partial} \boldsymbol{u}_{i}:=\int_{\mathcal{S}_{i}} \omega\left(\boldsymbol{r}_{i j}\right) \boldsymbol{K}_{i} \cdot \boldsymbol{p}_{i j} \otimes \boldsymbol{u}_{i j}^{T} \mathrm{~d} V_{j}$

For example, consider the displacement field $\boldsymbol{u}=(u, v)^{T}$ in two dimensional space, the relative displacement vector and the nonlocal partial derivatives have the explicit forms

$\boldsymbol{u}_{i j}=\left(\begin{array}{c}u_{j}-u_{i} \\ v_{j}-v_{i}\end{array}\right), \quad \tilde{\partial} \boldsymbol{u}_{i}=\left(\tilde{\partial} u_{i}, \tilde{\partial} v_{i}\right)=\left(\begin{array}{ll}u_{i, x} & v_{i, x} \\ u_{i, y} & v_{i, y} \\ u_{i, x x} & v_{i, x x} \\ u_{i, x y} & v_{i, x y} \\ u_{i, y y} & v_{i, y y}\end{array}\right)$,

Let $\boldsymbol{K}_{i} \cdot \boldsymbol{p}_{i j}$ be denoted by

$\left(g_{1 j}, g_{2 j}, h_{1 j}, h_{2 j}, h_{3 j}\right)^{T}=\boldsymbol{K}_{i} \cdot \boldsymbol{p}_{i j}$

The gradient vector $\boldsymbol{g}_{i j}$ and Hessian matrix $\boldsymbol{h}_{i j}$ between points $i$ and $j$ in 2D are, respectively

$\boldsymbol{g}_{i j}=\left(g_{1 j}, g_{2 j}\right)^{T}, \quad \boldsymbol{h}_{i j}=\left(\begin{array}{ll}h_{1 j} & h_{2 j} \\ h_{2 j} & h_{3 j}\end{array}\right)$ 
In 3D case, the polynomial vector based on relative coordinates $\boldsymbol{r}_{i j}=\left(x_{i j}, y_{i j}, z_{i j}\right)^{T}=\boldsymbol{x}_{j}-\boldsymbol{x}_{i}$ is given as

$\boldsymbol{p}_{i j}=\left(x_{i j}, y_{i j}, z_{i j}, x_{i j}^{2} / 2, x_{i j} y_{i j}, x_{i j} z_{i j}, y_{i j}^{2} / 2, y_{i j} z_{i j}, z_{i j}^{2}\right)^{T}$.

The shape tensor in 3D is constructed by Eq. 15 with $\boldsymbol{p}_{i j}$ in Eq. 23.

Let $\boldsymbol{K}_{i} \cdot \boldsymbol{p}_{i j}$ in 3D be denoted by

$\left(g_{1 j}, g_{2 j}, g_{3 j}, h_{1 j}, h_{2 j}, h_{3 j}, h_{4 j}, h_{5 j}, h_{6 j}\right)^{T}=\boldsymbol{K}_{i} \cdot \boldsymbol{p}_{i j}$.

The gradient vector $\boldsymbol{g}_{i j}$ and Hessian matrix $\boldsymbol{h}_{i j}$ for two points $i, j$ in support in 3D are, respectively

$\boldsymbol{g}_{i j}=\left(g_{1 j}, g_{2 j}, g_{3 j}\right)^{T}, \quad \boldsymbol{h}_{i j}=\left(\begin{array}{lll}h_{1 j} & h_{2 j} & h_{3 j} \\ h_{2 j} & h_{4 j} & h_{5 j} \\ h_{3 j} & h_{5 j} & h_{6 j}\end{array}\right)$

It is worth mentioning that for first order NOM or peridynamics, the gradient vector can be calculated as well by

$\boldsymbol{g}_{i j}=\left(\int_{\mathcal{S}_{i}} \omega\left(\boldsymbol{r}_{i k}\right) \boldsymbol{r}_{i k} \otimes \boldsymbol{r}_{i k} \mathrm{~d} V_{k}\right)^{-1} \cdot \boldsymbol{r}_{i j}$

Then the nonlocal gradient operator and Hessian operator for vector field can be defined as

$\tilde{\nabla} \otimes \boldsymbol{u}_{i}:=\int_{\mathcal{S}_{i}} \omega\left(\boldsymbol{r}_{i j}\right) \boldsymbol{u}_{i j} \otimes \boldsymbol{g}_{i j} \mathrm{~d} V_{j}$

$\tilde{\nabla} \otimes \tilde{\nabla} \otimes \boldsymbol{u}_{i}:=\int_{\mathcal{S}_{i}} \omega\left(\boldsymbol{r}_{i j}\right) \boldsymbol{u}_{i j} \otimes \boldsymbol{h}_{i j} \mathrm{~d} V_{j}$

In the case of 2-vector in 2 dimensional space, the explicit forms of $\tilde{\nabla} \otimes \boldsymbol{u}_{i}$ and $\tilde{\nabla} \otimes \tilde{\nabla} \otimes \boldsymbol{u}_{i}$ are

$\tilde{\nabla} \otimes \boldsymbol{u}_{i}=\left(\begin{array}{ll}u_{i, x} & u_{i, y} \\ v_{i, x} & v_{i, y}\end{array}\right)$

$\tilde{\nabla} \otimes \tilde{\nabla} \otimes \boldsymbol{u}_{i}=\left(\frac{\partial\left(\tilde{\nabla} \otimes \boldsymbol{u}_{i}\right)}{\partial x} \frac{\partial\left(\tilde{\nabla} \otimes \boldsymbol{u}_{i}\right)}{\partial y}\right)=\left(\left(\begin{array}{ll}u_{i, x x} & u_{i, y x} \\ v_{i, x x} & v_{i, y x}\end{array}\right)\left(\begin{array}{ll}u_{i, x y} & u_{i, y y} \\ v_{i, x y} & v_{i, y y}\end{array}\right)\right)$

For scalar-valued field, the nonlocal Laplace operator is the tensor contraction of $\tilde{\nabla} \otimes \tilde{\nabla} u_{i}$, e.g. $\tilde{\Delta}=\tilde{\nabla} \cdot \tilde{\nabla}=\operatorname{tr}(\tilde{\nabla} \otimes \tilde{\nabla})$, where $\operatorname{tr}(\cdot)$ denotes the trace of a matrix. More specifically, in $2 \mathrm{D}$

$\tilde{\Delta} u_{i}:=\int_{\mathcal{S}_{i}} \omega\left(\boldsymbol{r}_{i j}\right)\left(h_{1 j}+2 h_{2 j}+h_{3 j}\right) u_{i j} \mathrm{~d} V_{j}$

and in $3 \mathrm{D}$

$\tilde{\Delta} u_{i}:=\int_{\mathcal{S}_{i}} \omega\left(\boldsymbol{r}_{i j}\right)\left(h_{1 j}+2 h_{2 j}+2 h_{3 j}+h_{4 j}+2 h_{5 j}+h_{6 j}\right) u_{i j} \mathrm{~d} V_{j}$
And their local counterparts for scalar-valued field are

$\Delta w=w_{, y y}+2 w_{, x y}+w_{, x x} \quad$ in $2 \mathrm{D}$

$\Delta w=w_{, x x}+w_{, y y}+w_{, z z}+2 w_{, x y}+2 w_{, x z}+2 w_{, y z}$ in 3D .

\subsection{Stability of the second-order nonlocal operators}

According to Ref. [67], the energy functional for secondorder nonlocal operator in discrete form can be written as

$\mathcal{F}_{i}(\boldsymbol{u})=\frac{1}{2} \frac{p^{h g}}{m_{i}} \int_{\mathcal{S}_{i}} \omega\left(\boldsymbol{r}_{i j}\right)\left(u_{i j}-\boldsymbol{p}_{j}^{T} \tilde{\partial} u_{i}\right)^{2} \mathrm{~d} V_{j}$

where $p^{h g}$ is the penalty and $m_{i}=\int_{\mathcal{S}_{i}} \omega\left(\boldsymbol{r}_{i j}\right) \mathrm{d} V_{j}$. The operator in Eq. 14 corresponds to the minimum of Eq. 35. The first variation of $\mathcal{F}_{i}$ is

$$
\begin{aligned}
\delta \mathcal{F}_{i}(\boldsymbol{u}) & =\frac{p^{h g}}{m_{i}} \int_{\mathcal{S}_{i}} \omega\left(\boldsymbol{r}_{i j}\right)\left(u_{i j}-\boldsymbol{p}_{j}^{T} \tilde{\partial} u_{i}\right)\left(\delta u_{j}-\delta u_{i}-\boldsymbol{p}_{j}^{T} \tilde{\partial} \delta u_{i}\right) \mathrm{d} V_{j} \\
& =\frac{p^{h g}}{m_{i}} \int_{\mathcal{S}_{i}} \omega\left(\boldsymbol{r}_{i j}\right)\left(u_{i j}-\boldsymbol{p}_{j}^{T} \tilde{\partial} u_{i}\right)\left(\delta u_{j}-\delta u_{i}\right) \mathrm{d} V_{j} \\
& -\frac{p^{h g}}{m_{i}} \int_{\mathcal{S}_{i}} \omega\left(\boldsymbol{r}_{i j}\right)\left(u_{i j}-\boldsymbol{p}_{j}^{T} \tilde{\partial} u_{i}\right)\left(\boldsymbol{p}_{j}^{T} \tilde{\partial} \delta u_{i}\right) \mathrm{d} V_{j}
\end{aligned}
$$

We can prove that

$$
\begin{aligned}
& -\frac{p^{h g}}{m_{i}} \int_{\mathcal{S}_{i}} \omega\left(\boldsymbol{r}_{i j}\right)\left(u_{i j}-\boldsymbol{p}_{j}^{T} \tilde{\partial} u_{i}\right)\left(\boldsymbol{p}_{j}^{T} \tilde{\partial} \delta u_{i}\right) \mathrm{d} V_{j} \\
= & -\frac{p^{h g}}{m_{i}} \int_{j \in \mathcal{S}_{i}} \omega\left(\boldsymbol{r}_{i j}\right)\left(\boldsymbol{p}_{j} u_{i j}-\boldsymbol{p}_{j} \boldsymbol{p}_{j}^{T} \tilde{\partial} u_{i}\right) \mathrm{d} V_{j} \cdot \tilde{\partial} \delta u_{i} \\
= & -\frac{p^{h g}}{m_{i}}(\underbrace{\left.\int_{\mathcal{S}_{i}} \omega\left(\boldsymbol{r}_{i j}\right) \boldsymbol{p}_{j} u_{i j} \mathrm{~d} V_{j}-\int_{\mathcal{S}_{i}} \omega(\boldsymbol{r}) \boldsymbol{p}_{j} \boldsymbol{p}_{j}^{T} \mathrm{~d} V_{j} \cdot \tilde{\partial} u_{i}\right)}_{=0 \text { since Eq.13 }} \cdot \tilde{\partial} \delta u_{i}
\end{aligned}
$$

$=0$.

Therefore,

$\delta \mathcal{F}_{i}(\boldsymbol{u})=\frac{p^{h g}}{m_{i}} \int_{\mathcal{S}_{i}} \omega\left(\boldsymbol{r}_{i j}\right)\left(u_{i j}-\boldsymbol{p}_{j}^{T} \tilde{\partial} u_{i}\right)\left(\delta u_{j}-\delta u_{i}\right) \mathrm{d} V_{j}$

Consider integration of $\delta \mathcal{F}_{i}(\boldsymbol{u})$ in domain 


$$
\begin{aligned}
\int_{\Omega} \delta \mathcal{F}_{i} \mathrm{~d} V_{i} \\
=p^{h g} \underbrace{\int_{\Omega} \int_{\mathcal{S}_{i}} \frac{\omega\left(\boldsymbol{r}_{i j}\right)}{m_{i}}\left(u_{i j}-\boldsymbol{p}_{j}^{T} \tilde{\partial} u_{i}\right)\left(\delta u_{j}-\delta u_{i}\right) \mathrm{d} V_{j} \mathrm{~d} V_{i}}_{\text {by Eq.2 }} \\
=\int_{\Omega}\left(\int_{\mathcal{S}_{i}^{\prime}} \omega\left(\boldsymbol{r}_{i j}\right) \frac{p^{h g}}{m_{j}}\left(u_{j i}-\boldsymbol{p}_{i}^{T} \tilde{\partial} u_{j}\right) \mathrm{d} V_{j}\right. \\
\left.-\int_{\mathcal{S}_{i}} \omega(\boldsymbol{r}) \frac{p^{h g}}{m_{i}}\left(u_{i j}-\boldsymbol{p}_{j}^{T} \tilde{\partial} u_{i}\right) \mathrm{d} V_{j}\right) \delta u_{i} \mathrm{~d} V_{i} .
\end{aligned}
$$

For any $\delta u_{i}, \int_{\Omega} \delta \mathcal{F}_{i} \mathrm{~d} V_{i}=0$ leads to the internal force due to the stability of the nonlocal operator

$$
\begin{aligned}
& \int_{\mathcal{S}_{i}^{\prime}} \omega\left(\boldsymbol{r}_{i j}\right) \frac{p^{h g}}{m_{j}}\left(u_{j i}-\boldsymbol{p}_{i}^{T} \tilde{\partial} u_{j}\right) \mathrm{d} V_{j} \\
& -\int_{\mathcal{S}_{i}} \omega(\boldsymbol{r}) \frac{p^{h g}}{m_{i}}\left(u_{i j}-\boldsymbol{p}_{j}^{T} \tilde{\partial} u_{i}\right) \mathrm{d} V_{j}
\end{aligned}
$$

Equation 38 is the expression for a scalar-valued field. For vector-valued field, the internal force due to the stability of nonlocal operator is

$$
\begin{aligned}
& \int_{\mathcal{S}_{i}^{\prime}} \omega\left(\boldsymbol{r}_{j i}\right) \frac{p^{h g}}{m_{j}}\left(\boldsymbol{u}_{j i}-\boldsymbol{p}_{i}^{T} \tilde{\partial} \boldsymbol{u}_{j}\right) \mathrm{d} V_{j} \\
& -\int_{\mathcal{S}_{i}} \omega\left(\boldsymbol{r}_{i j}\right) \frac{p^{h g}}{m_{i}}\left(\boldsymbol{u}_{i j}-\boldsymbol{p}_{j}^{T} \tilde{\partial} \boldsymbol{u}_{i}\right) \mathrm{d} V_{j} .
\end{aligned}
$$

\section{Nonlocal governing equations based on NOM}

This section is devoted to the variational derivation of nonlocal strong forms of solid mechanics, including hyperelasticity, thin plate, gradient elasticity, electro-magnetic-elasticity theory and phase-field fracture method. The strong form is suitable for theoretical analysis as well as explicit time integration. For the fully implicit simulation of various PDEs, the reader is referred to NOM for PDEs [65-69, 72].

\subsection{Nonlocal form for hyperelasticity}

Consider the energy density of a hyperelasticity as $\psi:=\psi(\boldsymbol{F})$, where $\boldsymbol{F}=\nabla \boldsymbol{u}+\boldsymbol{I}$. The balance equation for the hyperelastic solid is

$\nabla \cdot \boldsymbol{P}+\boldsymbol{b}=0$ on $\Omega$

with boundary conditions $\boldsymbol{u}=\boldsymbol{u}_{0}$ on $\Gamma_{D}$ and $\boldsymbol{P} \cdot \boldsymbol{n}=\boldsymbol{t}_{0}$ on $\Gamma_{N}$, where $\boldsymbol{u}_{0}$ is the specified displacement and $\boldsymbol{t}_{0}$ is the prescribed traction load, $\boldsymbol{P}=\frac{\partial \psi}{\partial \boldsymbol{F}}$, the first Piola-Kirchhoff stress, $\boldsymbol{b}$ is the body force density.

\subsubsection{Derivation based on variational principle}

The variation of strain energy over the domain is

$$
\begin{aligned}
\delta \mathcal{F} & =\int_{\Omega} \delta \psi(\boldsymbol{F}) \mathrm{d} V=\int_{\Omega} \frac{\partial \psi}{\partial \boldsymbol{F}}: \delta \boldsymbol{F} \mathrm{d} V \\
& =\int_{\Omega} \boldsymbol{P}: \nabla(\delta \boldsymbol{u}) \mathrm{d} V \\
& =\int_{\Omega} \boldsymbol{P}_{i}: \int_{\mathcal{S}_{i}} \omega\left(\boldsymbol{r}_{i j}\right) \delta \boldsymbol{u}_{i j} \otimes \boldsymbol{g}_{i j} \mathrm{~d} V_{j} \mathrm{~d} V_{i} \\
& =\int_{\Omega} \int_{\mathcal{S}_{i}} \omega\left(\boldsymbol{r}_{i j}\right) \boldsymbol{P}_{i}: \delta \boldsymbol{u}_{i j} \otimes \boldsymbol{g}_{i j} \mathrm{~d} V_{j} \mathrm{~d} V_{i} \\
& =\int_{\Omega} \int_{\mathcal{S}_{i}} \omega\left(\boldsymbol{r}_{i j}\right)\left(\boldsymbol{P}_{i} \cdot \boldsymbol{g}_{i j}\right) \cdot \delta \boldsymbol{u}_{i j} \mathrm{~d} V_{j} \mathrm{~d} V_{i} \\
& =\underbrace{\int_{\mathcal{S}_{i}} \omega\left(\boldsymbol{r}_{i j}\right)\left(\boldsymbol{P}_{i} \cdot \boldsymbol{g}_{i j}\right) \cdot\left(\delta \boldsymbol{u}_{j}-\delta \boldsymbol{u}_{i}\right) \mathrm{d} V_{j} \mathrm{~d} V_{i}}_{\Omega} \\
& =\int_{\Omega}\left(\int_{\mathcal{S}_{i}^{\prime}} \omega\left(\boldsymbol{r}_{j i}\right) \boldsymbol{P}_{j} \cdot \boldsymbol{g}_{j i} \mathrm{~d} V_{j}-\int_{\mathcal{S}_{i}} \omega\left(\boldsymbol{r}_{i j}\right) \boldsymbol{P}_{i} \cdot \boldsymbol{g}_{i j} \mathrm{~d} V_{j}\right) \cdot \delta \boldsymbol{u}_{i} \mathrm{~d} V_{i}
\end{aligned}
$$

In above derivation, we replace the gradient operator with nonlocal gradient, e.g. $\tilde{\nabla} \otimes \boldsymbol{u}_{i} \rightarrow \int_{\mathcal{S}_{i}} \omega\left(\boldsymbol{r}_{i j}\right) \boldsymbol{u}_{i j} \otimes \boldsymbol{g}_{i j} \mathrm{~d} V_{j}$ in Eq. 27, and the relation $\boldsymbol{A}: \boldsymbol{a} \otimes \boldsymbol{b}=(\boldsymbol{A} \cdot \boldsymbol{b}) \cdot \boldsymbol{a}$ for secondorder tensor $\boldsymbol{A}$ and vectors $\boldsymbol{a}, \boldsymbol{b}$ is employed.

The variational of external body force energy

$\delta \mathcal{F}_{\text {ext }}=\int_{\Omega} \delta \boldsymbol{u} \cdot \boldsymbol{b} \mathrm{d} V$

For any $\delta \boldsymbol{u}_{i}, \delta \mathcal{F}-\delta \mathcal{F}_{\text {ext }}=0$ leads to the nonlocal governing equations for elasticity

$\int_{\mathcal{S}_{i}} \omega\left(\boldsymbol{r}_{i j}\right) \boldsymbol{P}_{i} \cdot \boldsymbol{g}_{i j} \mathrm{~d} V_{j}-\int_{\mathcal{S}_{i}^{\prime}} \omega\left(\boldsymbol{r}_{j i}\right) \boldsymbol{P}_{j} \cdot \boldsymbol{g}_{j i} \mathrm{~d} V_{j}+\boldsymbol{b}=0$

Considering the effect of inertial force $\rho \ddot{\boldsymbol{u}}_{i}$ per unit volume, and replacing the dual-support with dual-horizon, we obtain the equations of motion for dual-horizon peridynamics

$\int_{\mathcal{H}_{i}} \omega\left(\boldsymbol{r}_{i j}\right) \boldsymbol{P}_{i} \cdot \boldsymbol{g}_{i j} \mathrm{~d} V_{j}-\int_{\mathcal{H}_{i}^{\prime}} \omega\left(\boldsymbol{r}_{j i}\right) \boldsymbol{P}_{j} \cdot \boldsymbol{g}_{j i} \mathrm{~d} V_{j}+\boldsymbol{b}_{i}=\rho \ddot{\boldsymbol{u}} i$

If the sizes of horizons for all material points are the same, the dual-horizon peridynamics degenerates to the conventional constant horizon peridynamics.

For any specific strain energy density (for example, isotropic/anisotropic linear/nonlinear elasticity), the explicit form of $\boldsymbol{P}$ can be derived straightforwardly. In the section of numerical examples, we consider the linear isotropic 
elasticity, which can be viewed as a special case of the hyperelasticity.

\subsubsection{Derivation based on weighted residual method}

Beside the derivation based on strain energy density, the nonlocal strong form can be derived by weighted residual method. Consider the governing equations for hyperelasticity, the weak form of Eq. 40 for any trial vector becomes

$$
\begin{aligned}
0= & \int_{\Omega} \boldsymbol{v} \cdot \nabla \cdot \boldsymbol{P}+\boldsymbol{v} \cdot \boldsymbol{b} \mathrm{d} V \\
= & \int_{\Omega}-\nabla \boldsymbol{v}: \boldsymbol{P}+\boldsymbol{v} \cdot \boldsymbol{b} \mathrm{d} V+\int_{\Gamma} \boldsymbol{P} \cdot \boldsymbol{n} \cdot \boldsymbol{v} \mathrm{d} S \\
= & \int_{\Omega}-\left(\int_{\mathcal{S}_{i}} \omega\left(\boldsymbol{r}_{i j}\right) \boldsymbol{v}_{i j} \otimes \boldsymbol{g}_{i j} \mathrm{~d} V_{j}\right): \\
& \boldsymbol{P}_{i}+\boldsymbol{v}_{i} \cdot \boldsymbol{b} \mathrm{d} V_{i}+\int_{\Gamma} \boldsymbol{P} \cdot \boldsymbol{n} \cdot \boldsymbol{v} \mathrm{d} S
\end{aligned}
$$

Let us focus on the integral in $\Omega$, the first term in above equation can be written as

$$
\begin{aligned}
& \int_{\Omega}-\left(\int_{\mathcal{S}_{i}} \omega\left(\boldsymbol{r}_{i j}\right) \boldsymbol{v}_{i j} \otimes \boldsymbol{g}_{i j} \mathrm{~d} V_{j}\right): \boldsymbol{P}_{i} \mathrm{~d} V_{i} \\
& =\underbrace{\int_{\Omega}-\left(\int_{\mathcal{S}_{i}} \omega\left(\boldsymbol{r}_{i j}\right) \boldsymbol{P}_{i} \cdot \boldsymbol{g}_{i j} \cdot\left(\boldsymbol{v}_{j}-\boldsymbol{v}_{i}\right) \mathrm{d} V_{j}\right) \mathrm{d} V_{i}}_{\text {by Eq.2 }} \\
& =\int_{\Omega}\left(\int_{\mathcal{S}_{i}} \omega\left(\boldsymbol{r}_{i j}\right) \boldsymbol{P}_{i} \cdot \boldsymbol{g}_{i j} \mathrm{~d} V_{j}-\int_{\mathcal{S}_{i}^{\prime}} \omega\left(\boldsymbol{r}_{j i}\right) \boldsymbol{P}_{j} \cdot \boldsymbol{g}_{j i} \mathrm{~d} V_{j}\right) \cdot \boldsymbol{v}_{i} \mathrm{~d} V_{i}
\end{aligned}
$$

For any $\boldsymbol{v}_{i}$, the weak form being zero leads to

$$
\int_{\mathcal{S}_{i}} \omega\left(\boldsymbol{r}_{i j}\right) \boldsymbol{P}_{i} \cdot \boldsymbol{g}_{i j} \mathrm{~d} V_{j}-\int_{\mathcal{S}_{i}^{\prime}} \omega\left(\boldsymbol{r}_{j i}\right) \boldsymbol{P}_{j} \cdot \boldsymbol{g}_{j i} \mathrm{~d} V_{j}+\boldsymbol{b}=0
$$

which is identical to Eq. 43. As being more general than the energy method, the weighted residual method can be used to convert PDEs that have no energy functional to nonlocal integral forms.

\subsection{Nonlocal thin plate theory}

The thin plate theory is widely used in engineering applications [73]. The basic assumption of thin plate include: (1) the thickness of the plate is much smaller than the length inside the mid-plane; (2) the deflection is much smaller than the thickness of the plate so that higher-order effect is neglectable; (3) the stress along the thickness direction is assumed as zero, e.g. $\sigma_{z} \approx 0$ and the points in the midplane have no displacement parallel to the midplane, e.g. $u(x, y, 0)=v(x, y, 0) \approx 0 ;(4)$ the normal of the mid-plane remains perpendicular to the mid-plane after deformation.
Then the plate bending can be simplified into 2D problem and the displacements, strain and stress can be described by the deflection on the mid-plane

$u(x, y, z)=-z \frac{\partial w}{\partial x}$

$v(x, y, z)=-z \frac{\partial w}{\partial y}$

$w(x, y, z) \simeq w(x, y, 0) \cong w(x, y)$.

The generalized strain is the Hessian operator on the deflection

$\boldsymbol{\kappa}=\nabla^{2} w=\left(\begin{array}{ll}w_{, x x} & w_{, x y} \\ w_{, x y} & w_{, y y}\end{array}\right)$

with nonlocal correspondence and its variation

$\boldsymbol{\kappa}=\tilde{\nabla}^{2} w:=\int_{\mathcal{S}_{i}} \omega\left(\boldsymbol{r}_{i j}\right) \boldsymbol{h}_{i j} w_{i j} \mathrm{~d} V_{j}$

$\delta \boldsymbol{\kappa}=\int_{\mathcal{S}_{i}} \omega\left(\boldsymbol{r}_{i j}\right) \boldsymbol{h}_{i j} \delta w_{i j} \mathrm{~d} V_{j}$

The bending moment tensor $\boldsymbol{M}$, the general stress for isotropic thin plate, is given by

$\boldsymbol{M}=\left(\begin{array}{ll}M_{x x} & M_{x y} \\ M_{x y} & M_{y y}\end{array}\right)=D_{0}\left(v \operatorname{tr}(\boldsymbol{\kappa}) \boldsymbol{I}_{2 \times 2}+(1-v) \boldsymbol{\kappa}\right)$

where $D_{0}=\frac{E t^{3}}{12\left(1-v^{2}\right)}$ and $t$ is the thickness of the plate.

Based on the principle of minimum potential energy, the energy functional for the governing equation is

$\mathcal{F}_{\text {int }}=\int_{\Omega} \frac{1}{2} \boldsymbol{M}: \boldsymbol{\kappa}-q w \mathrm{~d} S$

and for the boundary condition can be expressed as

$\mathcal{F}_{\text {ext }}=\int_{S_{3}} \bar{V}_{n} w \mathrm{~d} \Gamma-\int_{S_{2}+S_{3}} \bar{M}_{n} \frac{\partial w}{\partial n} \mathrm{~d} \Gamma$

where $q$ is the external transverse load on the mid-plane, $\bar{V}_{n}$ is the shear force load on boundary $S_{3}$ and $\bar{M}_{n}$ is the prescribed moment on boundary $S_{2}+S_{3}$. For simplicity, we leave the integral on the boundary for later consideration. The variation of the internal energy functional is 


$$
\begin{aligned}
\delta \mathcal{F}_{i n t}= & \int_{\Omega} \boldsymbol{M}: \delta \boldsymbol{\kappa}-q \delta w \mathrm{~d} S \\
= & \int_{\Omega} \boldsymbol{M}_{i}: \int_{\mathcal{S}_{i}} \omega\left(\boldsymbol{r}_{i j}\right) \boldsymbol{h}_{i j} \delta w_{i j} \mathrm{~d} S_{j}-q_{i} \delta w_{i} \mathrm{~d} S_{i} \\
= & \underbrace{\int_{\Omega} \int_{\mathcal{S}_{i}} \omega\left(\boldsymbol{r}_{i j}\right) \boldsymbol{M}_{i}: \boldsymbol{h}_{i j}\left(\delta w_{j}-\delta w_{i}\right) \mathrm{d} S_{j}}_{\text {by Eq.2 }}-\int_{\Omega} q_{i} \delta w_{i} \mathrm{~d} S_{i} \\
= & \int_{\Omega}\left(\int_{\mathcal{S}_{i}^{\prime}} \omega\left(\boldsymbol{r}_{i j}\right) \boldsymbol{M}_{j}: \boldsymbol{h}_{j i} \mathrm{~d} S_{j}\right. \\
& \left.-\int_{\mathcal{S}_{i}} \omega\left(\boldsymbol{r}_{i j}\right) \boldsymbol{M}_{i}: \boldsymbol{h}_{i j} \mathrm{~d} S_{j}-q_{i}\right) \delta w_{i} \mathrm{~d} S_{i}
\end{aligned}
$$

The variation of the external energy function is

$$
\begin{aligned}
\delta \mathcal{F}_{e x t}= & \int_{S_{3}} \bar{V}_{n} \delta w \mathrm{~d} \Gamma-\int_{S_{2}+S_{3}} \bar{M}_{n} \frac{\partial \delta w}{\partial n} \mathrm{~d} \Gamma \\
= & \int_{S_{3}} \bar{V}_{n} \delta w \mathrm{~d} \Gamma-\int_{S_{2}+S_{3}} \bar{M}_{n} \nabla \delta w \cdot \boldsymbol{n} \mathrm{d} \Gamma \\
= & \int_{S_{3}} \bar{V}_{n} \delta w \mathrm{~d} \Gamma-\int_{S_{2}+S_{3}} \bar{M}_{n i} \\
& \int_{\mathcal{S}_{i}} \omega\left(\boldsymbol{r}_{i j}\right) \delta w_{i j} \boldsymbol{g}_{i j} \mathrm{~d} V_{j} \cdot \boldsymbol{n}_{i} \mathrm{~d} \Gamma_{i} \\
= & \int_{S_{3}} \bar{V}_{n} \delta w \mathrm{~d} \Gamma-\int_{S_{2}+S_{3}} \int_{\mathcal{S}_{i}} \omega\left(\boldsymbol{r}_{i j}\right) \\
& \bar{M}_{n i} \boldsymbol{g}_{i j} \cdot \boldsymbol{n}_{i} \delta w_{i j} \mathrm{~d} V_{j} \mathrm{~d} \Gamma_{i} \\
= & \int_{S_{3}} \bar{V}_{n} \delta w \mathrm{~d} \Gamma-\int_{S_{2}+S_{3}}\left(\int_{\mathcal{S}_{i}^{\prime}} \omega\left(\boldsymbol{r}_{j i}\right) \bar{M}_{n j} \boldsymbol{g}_{j i} \cdot \boldsymbol{n}_{j} \mathrm{~d} V_{j}\right. \\
& \left.-\int_{\mathcal{S}_{i}} \omega\left(\boldsymbol{r}_{i j}\right) \bar{M}_{n i} \boldsymbol{g}_{i j} \cdot \boldsymbol{n}_{i} \mathrm{~d} V_{j}\right) \delta w_{i} \mathrm{~d} \Gamma_{i}
\end{aligned}
$$

For any $\delta w_{i}, \delta \mathcal{F}_{\text {int }}-\delta \mathcal{F}_{\text {ext }}=0$ leads to the nonlocal thin plate equation for material point in domain $\Omega$

$$
\begin{aligned}
& \int_{\mathcal{S}_{i}} \omega\left(\boldsymbol{r}_{i j}\right) \boldsymbol{M}_{i}: \boldsymbol{h}_{i j} \mathrm{~d} V_{j} \\
& \quad-\int_{\mathcal{S}_{i}^{\prime}} \omega\left(\boldsymbol{r}_{i j}\right) \boldsymbol{M}_{j}: \boldsymbol{h}_{j i} \mathrm{~d} V_{j}+q_{i}=0
\end{aligned}
$$

The additional nonlocal form for material point applied with the moment boundary condition is

$$
\begin{aligned}
& \int_{\mathcal{S}_{i}} \omega\left(\boldsymbol{r}_{i j}\right) \bar{M}_{n i} \boldsymbol{g}_{i j} \cdot \boldsymbol{n}_{i} \mathrm{~d} V_{j} \\
& -\int_{\mathcal{S}_{i}^{\prime}} \omega\left(\boldsymbol{r}_{j i}\right) \bar{M}_{n j} \boldsymbol{g}_{j i} \cdot \boldsymbol{n}_{j} \mathrm{~d} V_{j}=0
\end{aligned}
$$

Based on the D'Alembert's principle, the equation of motion considering the effect of inertial force $\rho t \ddot{w}_{i}$ per unit area is

$$
\begin{aligned}
& \int_{\mathcal{S}_{i}^{\prime}} \omega\left(\boldsymbol{r}_{i j}\right) \boldsymbol{M}_{j}: \boldsymbol{h}_{j i} \mathrm{~d} V_{j} \\
& \quad-\int_{\mathcal{S}_{i}} \omega\left(\boldsymbol{r}_{i j}\right) \boldsymbol{M}_{i}: \boldsymbol{h}_{i j} \mathrm{~d} V_{j}+q_{i}=t \rho \ddot{w}_{i}
\end{aligned}
$$

For clamped boundary condition $w_{, n}=\nabla w \cdot \boldsymbol{n}=0$, the nonlocal form is

$\int_{\mathcal{S}_{i}} \omega\left(\boldsymbol{r}_{i j}\right) w_{i j} \boldsymbol{g}_{i j} \cdot \boldsymbol{n}_{i} \mathrm{~d} V_{j}=0$

Compared with the local governing equation for thin plate $\nabla^{2}: \boldsymbol{M}+q=t \rho \ddot{w}$, we can find the correspondence between local and nonlocal formulation

$$
\begin{aligned}
\nabla^{2} & : \boldsymbol{M} \rightarrow \tilde{\nabla}^{2}: \boldsymbol{M}_{i}:=\int_{\mathcal{S}_{i}^{\prime}} \omega\left(\boldsymbol{r}_{j i}\right) \boldsymbol{M}_{j}: \boldsymbol{h}_{j i} \mathrm{~d} V_{j} \\
& -\int_{\mathcal{S}_{i}} \omega\left(\boldsymbol{r}_{i j}\right) \boldsymbol{M}_{i}: \boldsymbol{h}_{i j} \mathrm{~d} V_{j}
\end{aligned}
$$

The nonlocal derivation for thin plate can be extended to composite plate and functional gradient plate theories.

\subsection{Nonlocal gradient elasticity}

Gradient theories emerge from considerations of the microstructure in the material at micro-scale, where a mass point after homogenization is not the center of a micro-volume and the rotation of the micro-volume depends on the moment stress/couple stress as well as the Cauchy stress. Gradient elasticity generalizes the elasticity theory by employing higher order terms of the deformation gradient or the gradient of the strain tensor. Generally, the energy density functional can be assumed as $\psi:=\psi(\boldsymbol{F}, \nabla \boldsymbol{F})=\psi\left(\nabla \boldsymbol{u}, \nabla^{2} \boldsymbol{u}\right)$, where $\boldsymbol{F}=\nabla \boldsymbol{u}+\boldsymbol{I}$. The total potential energy in domain is

$\mathcal{F}=\int_{\Omega} \psi-\boldsymbol{b} \cdot \boldsymbol{u} \mathrm{d} V$

The stress tensor and generalized stress tensor of first PiolaKirchhoff type are defined as

$\boldsymbol{P}=\frac{\partial \psi}{\partial \boldsymbol{F}}$

$\boldsymbol{\Sigma}=\frac{\partial \psi}{\partial \nabla \boldsymbol{F}}$

The variation of the total internal energy is 


$$
\begin{aligned}
\delta \mathcal{F}= & \int_{\Omega}\left(\frac{\partial \psi}{\partial \boldsymbol{F}}: \nabla \delta \boldsymbol{u}\right. \\
& \left.+\frac{\partial \psi}{\partial \nabla \boldsymbol{F}}: \nabla^{2} \delta \boldsymbol{u}-\boldsymbol{b} \cdot \delta \boldsymbol{u}\right) \mathrm{d} V \\
= & \int_{\Omega}(\boldsymbol{P}: \nabla \delta \boldsymbol{u} \\
& \left.+\boldsymbol{\Sigma}: \nabla^{2} \delta \boldsymbol{u}-\boldsymbol{b} \cdot \delta \boldsymbol{u}\right) \mathrm{d} V
\end{aligned}
$$

Based on the integration by parts, the local form can be derived by

$$
\begin{aligned}
\delta \mathcal{F}= & \int_{\partial \Omega}(\boldsymbol{n} \cdot \boldsymbol{P} \cdot \delta \boldsymbol{u}+\boldsymbol{n} \cdot \boldsymbol{\Sigma}: \nabla \delta \boldsymbol{u}) \mathrm{d} S \\
& -\int_{\Omega}(\nabla \cdot \boldsymbol{P} \cdot \delta \boldsymbol{u}+\nabla \cdot \boldsymbol{\Sigma}: \nabla \delta \boldsymbol{u}+\boldsymbol{b} \cdot \boldsymbol{u}) \mathrm{d} V \\
= & \int_{\partial \Omega}(\boldsymbol{n} \cdot \boldsymbol{P} \cdot \delta \boldsymbol{u}+\boldsymbol{n} \cdot \boldsymbol{\Sigma}: \nabla \delta \boldsymbol{u}-\boldsymbol{n} \cdot \nabla \cdot \boldsymbol{\Sigma} \cdot \delta \boldsymbol{u}) \mathrm{d} S \\
& -\int_{\Omega}\left(\nabla \cdot \boldsymbol{P}-\nabla^{2}: \boldsymbol{\Sigma}+\boldsymbol{b}\right) \cdot \delta \boldsymbol{u} \mathrm{d} . V
\end{aligned}
$$

Based on D'Alembert's principle, the governing equations for dynamic gradient elasticity can be written as

$\nabla \cdot \boldsymbol{P}-\nabla^{2}: \boldsymbol{\Sigma}+\boldsymbol{b}=\rho \ddot{\boldsymbol{u}}$ in $\Omega$.

On the other hand, do the substitutions $\nabla \delta \boldsymbol{u} \rightarrow \int_{\mathcal{S}_{i}} \omega\left(\boldsymbol{r}_{i j}\right) \boldsymbol{g}_{i j} \otimes \delta \boldsymbol{u}_{i j} \mathrm{~d} V_{j}$, and $\nabla^{2} \delta \boldsymbol{u} \rightarrow \int_{\mathcal{S}_{i}} \omega\left(\boldsymbol{r}_{i j}\right) \boldsymbol{h}_{i j} \otimes \delta \boldsymbol{u}_{i j} \mathrm{~d} V_{j}$ we get

$$
\begin{aligned}
\delta \mathcal{F}= & \int_{\Omega} \boldsymbol{P}: \nabla \delta \boldsymbol{u}+\boldsymbol{\Sigma}: \nabla^{2} \delta \boldsymbol{u}-\boldsymbol{b} \cdot \delta \boldsymbol{u} \mathrm{d} V \\
= & \int_{\Omega}\left(\boldsymbol{P}_{i}: \int_{\mathcal{S}_{i}} \omega\left(\boldsymbol{r}_{i j}\right) \boldsymbol{g}_{i j} \otimes \delta \boldsymbol{u}_{i j} \mathrm{~d} V_{j}\right. \\
& \left.+\boldsymbol{\Sigma}_{i}: \int_{\mathcal{S}_{i}} \omega\left(\boldsymbol{r}_{i j}\right) \boldsymbol{h}_{i j} \otimes \delta \boldsymbol{u}_{i j} \mathrm{~d} V_{j}-\boldsymbol{b} \cdot \delta \boldsymbol{u}\right) \mathrm{d} V_{i} \\
= & \underbrace{\int_{\Omega} \omega\left(\boldsymbol{r}_{i j}\right) \boldsymbol{P}_{i}:\left(\delta \boldsymbol{u}_{j}-\delta \boldsymbol{u}_{i}\right) \otimes \boldsymbol{g}_{i j} \mathrm{~d} V_{j} \mathrm{~d} V_{i}}_{\text {by Eq.2 }} \\
& +\underbrace{\int_{\Omega} \int_{\mathcal{S}_{i}} \omega\left(\boldsymbol{r}_{i j}\right) \boldsymbol{\Sigma}_{i}:\left(\delta \boldsymbol{u}_{j}-\delta \boldsymbol{u}_{j}\right) \otimes \boldsymbol{h}_{i j} \mathrm{~d} V_{j} \mathrm{~d} V_{i}-\int_{\Omega} \boldsymbol{b} \cdot \delta \boldsymbol{u}_{i} \mathrm{~d} V_{i}}_{\text {by Eq.2 }} \\
= & \int_{\Omega}\left(\int_{\mathcal{S}_{i}^{\prime}} \omega\left(\boldsymbol{r}_{j i}\right) \boldsymbol{P}_{j} \cdot \boldsymbol{g}_{j i} \mathrm{~d} V_{j}\right. \\
& \left.-\int_{\mathcal{S}_{i}} \omega\left(\boldsymbol{r}_{i j}\right) \boldsymbol{P}_{i} \cdot \boldsymbol{g}_{i j} \mathrm{~d} V_{j}\right) \cdot \delta \boldsymbol{u}_{i} \mathrm{~d} V_{i} \\
& +\int_{\Omega}\left(\int_{\mathcal{S}_{i}^{\prime}} \omega\left(\boldsymbol{r}_{j i}\right) \boldsymbol{\Sigma}_{j}: \boldsymbol{h}_{j i} \mathrm{~d} V_{j}\right. \\
& \left.-\int_{\mathcal{S}_{i}} \omega\left(\boldsymbol{r}_{i j}\right) \boldsymbol{\Sigma}_{i}: \boldsymbol{h}_{i j} \mathrm{~d} V_{j}\right) \cdot \delta \boldsymbol{u}_{i} \mathrm{~d} V_{i} \\
& -\int_{\Omega} \boldsymbol{b} \cdot \delta \boldsymbol{u}_{i} \mathrm{~d} V_{i} .
\end{aligned}
$$

In the above derivation, we used $\boldsymbol{\Sigma}: \boldsymbol{u} \otimes \boldsymbol{h}=(\boldsymbol{\Sigma}: \boldsymbol{h}) \cdot \boldsymbol{u}$. For any $\delta \boldsymbol{u}_{i}, \delta \mathcal{F}=0$ leads to the nonlocal form of gradient elasticity

$$
\begin{aligned}
& \int_{\mathcal{S}_{i}} \omega\left(\boldsymbol{r}_{i j}\right)\left(\boldsymbol{P}_{i} \cdot \boldsymbol{g}_{i j}+\boldsymbol{\Sigma}_{i}: \boldsymbol{h}_{i j}\right) \mathrm{d} V_{j} \\
& \quad-\int_{\mathcal{S}_{i}^{\prime}} \omega\left(\boldsymbol{r}_{j i}\right)\left(\boldsymbol{P}_{j} \cdot \boldsymbol{g}_{j i}+\boldsymbol{\Sigma}_{j}: \boldsymbol{h}_{j i}\right) \mathrm{d} V_{j}+\boldsymbol{b}=\rho \ddot{\boldsymbol{u}}_{i} .
\end{aligned}
$$

The inertia force term is added based on D'Alembert's principle.

Comparing Eqs. 67 and 69, the correspondence from local form to nonlocal form is

$$
\begin{gathered}
\nabla^{2}: \boldsymbol{\Sigma}_{i} \rightarrow \int_{\mathcal{S}_{i}^{\prime}} \omega\left(\boldsymbol{r}_{j i}\right) \boldsymbol{\Sigma}_{j}: \boldsymbol{h}_{j i} \mathrm{~d} V_{j} \\
-\int_{\mathcal{S}_{i}} \omega\left(\boldsymbol{r}_{i j}\right) \boldsymbol{\Sigma}_{i}: \boldsymbol{h}_{i j} \mathrm{~d} V_{j}
\end{gathered}
$$

\subsection{Nonlocal form of magneto-electro-elasticity}

In accordance with reference [74], let us postulate the following form of internal energy for the energy function $\psi:=\psi(\boldsymbol{F}, \nabla \boldsymbol{F}, \boldsymbol{p}, \nabla \boldsymbol{p}, \boldsymbol{m}, \nabla \boldsymbol{m})$, a function depends on the displacement gradient $\boldsymbol{F}=\nabla \boldsymbol{u}+\boldsymbol{I}$ and its second gradient $\nabla \boldsymbol{F}=\nabla^{2} \boldsymbol{u}$, polarization vector $\boldsymbol{p}$ and its gradient $\nabla \boldsymbol{p}$, magnetic field $\boldsymbol{m}$ and its gradient $\nabla \boldsymbol{m}$. The total potential energy in the domain can be written as

$\mathcal{F}=\int_{\Omega} \psi(\boldsymbol{F}, \nabla \boldsymbol{F}, \boldsymbol{p}, \nabla \boldsymbol{p}, \boldsymbol{m}, \nabla \boldsymbol{m}) \mathrm{d} V$

This model has a strong physical background, for example, the nonlinear electro-gradient elasticity for semiconductors [75] and flexoelectricity [76].

The first variation of $\mathcal{F}$ is

$$
\begin{aligned}
\delta \mathcal{F}= & \int_{\Omega} \delta \psi \mathrm{d} V \\
= & \int_{\Omega} \frac{\partial \psi}{\partial \boldsymbol{F}}: \nabla \delta \boldsymbol{u}+\frac{\partial \psi}{\partial \nabla \boldsymbol{F}}: \nabla^{2} \delta \boldsymbol{u}+\frac{\partial \psi}{\partial \boldsymbol{p}} \cdot \delta \boldsymbol{p}+ \\
& \frac{\partial \psi}{\partial \nabla \boldsymbol{p}}: \nabla \delta \boldsymbol{p}+\frac{\partial \psi}{\partial \boldsymbol{m}} \cdot \delta \boldsymbol{m}+\frac{\partial \psi}{\partial \nabla \boldsymbol{m}}: \nabla \delta \boldsymbol{m} \mathrm{d} V \\
= & \int_{\Omega} \boldsymbol{P}: \nabla \delta \boldsymbol{u}+\boldsymbol{\Sigma}: \nabla^{2} \delta \boldsymbol{u}+\boldsymbol{e} \cdot \delta \boldsymbol{p} \\
& +\boldsymbol{E}: \nabla \delta \boldsymbol{p}+\boldsymbol{s} \cdot \delta \boldsymbol{m}+\boldsymbol{S}: \nabla \delta \boldsymbol{m} \mathrm{d} V
\end{aligned}
$$

where

$$
\begin{aligned}
& \boldsymbol{P}=\frac{\partial \psi}{\partial \boldsymbol{F}}, \boldsymbol{\Sigma}=\frac{\partial \psi}{\partial \nabla \boldsymbol{F}}, \boldsymbol{e}=\frac{\partial \psi}{\partial \boldsymbol{p}} \\
& \boldsymbol{E}=\frac{\partial \psi}{\partial \nabla \boldsymbol{p}}, \boldsymbol{s}=\frac{\partial \psi}{\partial \boldsymbol{m}}, \boldsymbol{S}=\frac{\partial \psi}{\partial \nabla \boldsymbol{m}}
\end{aligned}
$$


Doing substitutions $\nabla \delta \boldsymbol{u}_{i} \rightarrow \int_{\mathcal{S}_{i}} \omega\left(\boldsymbol{r}_{i j}\right) \delta \boldsymbol{u}_{i j} \otimes \boldsymbol{g}_{i j} \mathrm{~d} V_{j}$, $\nabla^{2} \delta \boldsymbol{u}_{i} \rightarrow \int_{\mathcal{S}_{i}} \omega\left(\boldsymbol{r}_{i j}\right) \delta \boldsymbol{u}_{i j} \otimes \boldsymbol{h}_{i j} \mathrm{~d} V_{j}$

$\nabla \delta \boldsymbol{p}_{i} \rightarrow \int_{\mathcal{S}_{i}} \omega\left(\boldsymbol{r}_{i j}\right) \delta \boldsymbol{p}_{i j} \otimes \boldsymbol{g}_{i j} \mathrm{~d} V_{j}$

,$\nabla \delta \boldsymbol{m}_{i} \rightarrow \int_{\mathcal{S}_{i}} \omega\left(\boldsymbol{r}_{i j}\right) \delta \boldsymbol{m}_{i j} \otimes \boldsymbol{g}_{i j} \mathrm{~d} V_{j}$ and following the same operations in prior sections, the functional becomes

$$
\begin{aligned}
\delta \mathcal{F}= & \int_{\Omega}\left(\int_{\mathcal{S}_{i}^{\prime}} \omega\left(\boldsymbol{r}_{j i}\right)\left(\boldsymbol{P}_{j} \cdot \boldsymbol{g}_{j i}+\boldsymbol{\Sigma}_{j}: \boldsymbol{h}_{j i}\right) \mathrm{d} V_{j}\right. \\
& \left.-\int_{\mathcal{S}_{i}} \omega\left(\boldsymbol{r}_{i j}\right)\left(\boldsymbol{P}_{i} \cdot \boldsymbol{g}_{i j}+\boldsymbol{\Sigma}_{i}: \boldsymbol{h}_{i j}\right) \mathrm{d} V_{j}\right) \cdot \delta \boldsymbol{u}_{i} \mathrm{~d} V_{i} \\
+ & \int_{\Omega}\left(\int_{\mathcal{S}_{i}^{\prime}} \omega\left(\boldsymbol{r}_{j i}\right)\left(\boldsymbol{E}_{j} \cdot \boldsymbol{g}_{j i}\right) \mathrm{d} V_{j}\right. \\
& \left.-\int_{\mathcal{S}_{i}} \omega\left(\boldsymbol{r}_{i j}\right) \boldsymbol{E}_{i} \cdot \boldsymbol{g}_{i j} \mathrm{~d} V_{j}+\boldsymbol{e}_{i}\right) \cdot \delta \boldsymbol{p}_{i} \mathrm{~d} V_{i}+ \\
\int_{\Omega} & \left(\int_{\mathcal{S}_{i}^{\prime}} \omega\left(\boldsymbol{r}_{j i}\right)\left(\boldsymbol{S}_{j} \cdot \boldsymbol{g}_{j i}\right) \mathrm{d} V_{j}\right. \\
& \left.-\int_{\mathcal{S}_{i}} \omega\left(\boldsymbol{r}_{i j}\right) \boldsymbol{S}_{i} \cdot \boldsymbol{g}_{i j} \mathrm{~d} V_{j}+\boldsymbol{s}_{i}\right) \cdot \delta \boldsymbol{m}_{i} \mathrm{~d} V_{i}
\end{aligned}
$$

For any $\delta \boldsymbol{u}_{i}, \delta \boldsymbol{p}_{i}, \delta \boldsymbol{m}_{i}, \delta \mathcal{F}=0$ leads to general nonlocal governing equation for mechanical field, electrical field and magnetic field, respectively

$$
\begin{aligned}
& \int_{\mathcal{S}_{i}} \omega\left(\boldsymbol{r}_{i j}\right)\left(\boldsymbol{P}_{i} \cdot \boldsymbol{g}_{i j}+\boldsymbol{\Sigma}_{i}: \boldsymbol{h}_{i j}\right) \mathrm{d} V_{j} \\
& -\int_{\mathcal{S}_{i}^{\prime}} \omega\left(\boldsymbol{r}_{j i}\right)\left(\boldsymbol{P}_{j} \cdot \boldsymbol{g}_{j i}+\boldsymbol{\Sigma}_{j}: \boldsymbol{h}_{j i}\right) \mathrm{d} V_{j}+\boldsymbol{b}_{i}=0 \\
& \int_{\mathcal{S}_{i}} \omega\left(\boldsymbol{r}_{i j}\right) \boldsymbol{E}_{i} \cdot \boldsymbol{g}_{i j} \mathrm{~d} V_{j} \\
& -\int_{\mathcal{S}_{i}^{\prime}} \omega\left(\boldsymbol{r}_{j i}\right) \boldsymbol{E}_{j} \cdot \boldsymbol{g}_{j i} \mathrm{~d} V_{j}-\boldsymbol{e}_{i}=0 \\
& \int_{\mathcal{S}_{i}} \omega\left(\boldsymbol{r}_{i j}\right) \boldsymbol{S}_{i} \cdot \boldsymbol{g}_{i j} \mathrm{~d} V_{j} \\
& \quad-\int_{\mathcal{S}_{i}^{\prime}} \omega\left(\boldsymbol{r}_{j i}\right) \boldsymbol{S}_{j} \cdot \boldsymbol{g}_{j i} \mathrm{~d} V_{j}-\boldsymbol{s}_{i}=0
\end{aligned}
$$

In the derivation, we did not specify the exact form of the energy density, whether it is of small deformation or of finite deformation. For the specified energy form, one only needs to derive the expression for $\boldsymbol{P}, \boldsymbol{\Sigma}, \boldsymbol{e}, \boldsymbol{E}, \boldsymbol{s}, \boldsymbol{S}$ based on the material constitutions. It can be seen that the nonlocal governing equations for the continuum magneto-electro-elasticity can be obtained with ease by using nonlocal operator method and variational principle. The same rule applies for many other physical problems.

\subsection{Nonlocal form of phase-field fracture method}

Phase-field fracture method is powerful in fracture modelling [77]. The difference in tensile and compressive strengths of the material can be considered by dividing the strain energy density into a tensile part affected by the phase field and a compressive part, which is independent of the phase field,

$\psi_{e}(\boldsymbol{\varepsilon}(\nabla \boldsymbol{u}), s)=(1-s)^{2} \psi_{e}^{+}(\varepsilon(\nabla \boldsymbol{u}))+\psi_{e}^{-}(\varepsilon(\nabla \boldsymbol{u}))$.

where $\psi_{e}^{+}\left(\psi_{e}^{-}\right)$denotes the strain energy density for tensile (compressive) part, $\boldsymbol{u}$ is the displacement, $s \in[0,1]$ is the phase field, $\varepsilon$ denotes the strain and $\ell$ is the phase-field intrinsic length scale.

The full potential functional of the phase-field fracture model reads

$$
\begin{aligned}
\mathcal{F}_{\ell}(\boldsymbol{u}, s)= & \int_{\Omega}\left((1-s)^{2} \psi_{e}^{+}(\boldsymbol{\varepsilon}(\nabla \boldsymbol{u}))+\psi_{e}^{-}(\boldsymbol{\varepsilon}(\nabla \boldsymbol{u}))\right) \mathrm{d} V \\
& -\int_{\partial \Omega} \boldsymbol{t}^{*} \cdot \boldsymbol{u} \mathrm{d} A-\int_{\Omega} \boldsymbol{b} \cdot \boldsymbol{u} \mathrm{d} V+\int_{\Omega} g_{c}\left(\frac{s^{2}}{2 \ell}+\frac{\ell}{2} \nabla s \cdot \nabla s\right) \mathrm{d} V,
\end{aligned}
$$

where $\boldsymbol{t}^{*}$ denotes the surface traction at the boundary, $\boldsymbol{b}$ is the body force density and $g_{c}$ is the critical energy release rate.

For the sake of simplicity, we neglect the surface traction force and consider the first variation of $\mathcal{F}_{\ell}$ 


$$
\begin{aligned}
\delta \mathcal{F}_{\ell}= & \int_{\Omega} \delta\left((1-s)^{2} \psi_{e}^{+}+\psi_{e}^{-}\right) \mathrm{d} V-\int_{\Omega} \boldsymbol{b} \cdot \delta \boldsymbol{u} \mathrm{d} V & \int_{\mathcal{S}_{i}} \omega\left(\boldsymbol{r}_{i j}\right) \boldsymbol{\sigma}_{i} \cdot \boldsymbol{g}_{i j} \mathrm{~d} V_{j} \\
& +\int_{\Omega} g_{c} \delta\left(\frac{s^{2}}{2 \ell}+\frac{\ell}{2} \nabla s \cdot \nabla s\right) \mathrm{d} V & -\int_{\mathcal{S}_{i}^{\prime}} \omega\left(\boldsymbol{r}_{j i}\right) \boldsymbol{\sigma}_{j} \cdot \boldsymbol{g}_{j i} \mathrm{~d} V_{j}+\boldsymbol{b}_{i}=0 \\
= & \int_{\Omega}\left((1-s)^{2} \frac{\partial \psi_{e}^{+}}{\partial \boldsymbol{\varepsilon}}: \nabla \delta \boldsymbol{u}\right. & \\
& \left.-2 \psi_{e}^{+}(1-s) \delta s+\frac{\partial \psi_{e}^{-}}{\partial \boldsymbol{\varepsilon}}: \nabla \delta \boldsymbol{u}\right) \mathrm{d} V-\int_{\Omega} \boldsymbol{b} \cdot \delta \boldsymbol{u} \mathrm{d} V & \frac{s_{i}}{\ell}-2 \frac{\psi_{e i}^{+}}{g_{c}}\left(1-s_{i}\right)+\int_{\mathcal{S}_{i}^{\prime}} \omega\left(\boldsymbol{r}_{j i}\right) \ell \nabla s_{j} \cdot \boldsymbol{g}_{j i} \mathrm{~d} V_{j} \\
+ & \int_{\Omega} g_{c}\left(\frac{s}{\ell} \delta s+\ell \nabla s \cdot \nabla \delta s\right) \mathrm{d} V & -\int_{\mathcal{S}_{i}} \omega\left(\boldsymbol{r}_{i j}\right) \ell \nabla s_{i} \cdot \boldsymbol{g}_{i j} \mathrm{~d} V_{j}=0 .
\end{aligned}
$$$$
=\int_{\Omega}\left(\left((1-s)^{2} \boldsymbol{\sigma}^{+}+\boldsymbol{\sigma}^{-}\right): \nabla \delta \boldsymbol{u}-\boldsymbol{b} \cdot \delta \boldsymbol{u}\right) \mathrm{d} V
$$$$
+\int_{\Omega} g_{c}\left(\frac{s}{\ell} \delta s-2 \frac{\psi_{e}^{+}}{g_{c}}(1-s) \delta s+\ell \nabla s \cdot \nabla \delta s\right) \mathrm{d} V
$$$$
=\int_{\Omega}\left(\boldsymbol{\sigma}_{i}: \nabla \delta \boldsymbol{u}_{i}-\boldsymbol{b}_{i} \cdot \delta \boldsymbol{u}_{i}\right) \mathrm{d} V_{i}
$$$$
+\int_{\Omega} g_{c}\left(\frac{s_{i}}{\ell} \delta s_{i}-2 \frac{\psi_{e i}^{+}}{g_{c}}\left(1-s_{i}\right) \delta s_{i}+\ell \nabla s_{i} \cdot \nabla \delta s_{i}\right) \mathrm{d} V_{i}
$$$$
=\int_{\Omega}\left(\sigma_{i}:\left(\int_{\mathcal{S}_{i}} \omega\left(\boldsymbol{r}_{i j}\right) \delta \boldsymbol{u}_{i j} \otimes g_{i j} \mathrm{~d} V_{j}\right)\right.
$$$$
\left.-\boldsymbol{b}_{i} \cdot \delta \boldsymbol{u}_{i}\right) \mathrm{d} V_{i}
$$$$
+\int_{\Omega} g_{c}\left(\frac{s_{i}}{\ell} \delta s_{i}-2 \frac{\psi_{e i}^{+}}{g_{c}}\left(1-s_{i}\right) \delta s_{i}+\ell \nabla s_{i} .\right.
$$$$
\left.\int_{\mathcal{S}_{i}} \omega\left(\boldsymbol{r}_{i j}\right) \delta s_{i j} \boldsymbol{g}_{i j} \mathrm{~d} V_{j}\right) \mathrm{d} V_{i}
$$$$
=\int_{\Omega}\left(\left(\int_{\mathcal{S}_{i}^{\prime}} \omega\left(\boldsymbol{r}_{j i}\right) \boldsymbol{\sigma}_{j} \cdot \boldsymbol{g}_{j i} \mathrm{~d} V_{j}\right.\right.
$$$$
\left.\left.-\int_{\mathcal{S}_{i}} \omega\left(\boldsymbol{r}_{i j}\right) \boldsymbol{\sigma}_{i} \cdot \boldsymbol{g}_{i j} \mathrm{~d} V_{j}\right) \cdot \delta \boldsymbol{u}_{i}-\boldsymbol{b}_{i} \cdot \delta \boldsymbol{u}_{i}\right) \mathrm{d} V_{i}
$$$$
+\int_{\Omega} g_{c}\left(\frac{s_{i}}{\ell}-2 \frac{\psi_{e i}^{+}}{g_{c}}\left(1-s_{i}\right)+\int_{\mathcal{S}_{i}^{\prime}} \omega\left(\boldsymbol{r}_{j i}\right) \ell \nabla s_{j} \cdot \boldsymbol{g}_{j i} \mathrm{~d} V_{j}\right.
$$$$
\left.-\int_{\mathcal{S}_{i}} \omega\left(\boldsymbol{r}_{i j}\right) \ell \nabla s_{i} \cdot \boldsymbol{g}_{i j} \mathrm{~d} V_{j}\right) \delta s_{i} \mathrm{~d} V_{i}
$$

where

$$
\begin{aligned}
& \sigma^{+}=\frac{\partial \psi_{e}^{+}}{\partial \varepsilon}, \sigma^{-}=\frac{\partial \psi_{e}^{-}}{\partial \varepsilon} \\
& \sigma=(1-s)^{2} \sigma^{+}+\sigma^{-}
\end{aligned}
$$

For any $\delta \boldsymbol{u}_{i}, \delta s_{i}, \delta \mathcal{F}_{\ell}=0$ leads to the nonlocal governing equations for the mechanical field and phase field
The above examples aim at illustrating the power of nonlocal operator method combined with weighted residual method or variational principle in the derivation of nonlocal strong forms based on their local strong or energy forms. The derived nonlocal strong forms are variationally consistent and allow variable support sizes for each point in the model.

\section{Instability criterion for fracture modelling}

Typical methods for fracture modelling are either based on diffusive crack domain in phase-field methods or on direct topological modification on meshes in XFEM or bonds in PD. Direct topological modification on meshes often leads to instability issues. For example, in NOSBPD, the breakage of a bond based on the quantities derived from stress state or strain state often introduces too much perturbation to the scheme, which may abort the calculation because of the singularity in shape tensors. These criteria include critical stretch [29], energy based [31] or stress-based criterion $[33,34]$. Another issue in NOSBPD is that the strain energy carried by a bond is closely related to other bonds. It also depends on the direction, the length of the bond, the choice of influence functions. Removing one neighbour often gives rise to catastrophic results on the calculation. A criterion on how to remove the neighbours safely from the neighbour list remains unclear.

Damage is a process deviated from the robust mathematical expression, where the transition happens in a very narrow zone, such as the crack tip front. It is observed that around the crack tip, the gradient or strain undergoes a sharp transition within a very small zone. Most conventional numerical methods for fracture modelling focus on accurate description of the singularity occurring around the crack tip, such a description is very hard to tackle and its evolution is inconvenient to update. This dilemma can be handled when something different from continuous function is introduced.

In NOM, the gradient operator is defined in a "redundant" way. Around the crack tip, the deformation is irregular and the part due to hourglass energy is comparable to the strain energy carried by a particle. More specifically, the operator 
energy in nonlocal operator method describes the irregularity of a function around the crack tip. The irregularity is the part that cannot be described by the continuous function. For continuous domain, the strain energy density is much larger than the operator energy density. However, for particles around the crack tip, the operator energy density is far from zero and the irregularity due to the singularity around the crack tip increases comparably to the strain energy density. In this sense, the operator energy density can be viewed as an indicator for the crack tip.

Unlike the strain energy density, the hourglass energy density describes the irregular deformation around the crack tip. It depends on the penalty for the strain energy. Larger penalty improves the continuity of deformation, but the extent of hourglass energy compared with the strain energy density is hard to estimate. In this paper, we propose a special manner to estimate the critical hourglass strain. Let the critical bond strain be denoted by $s_{\max }$, which may depend on the characteristic length scale of the support, critical energy release rate and the elastic modulus. When the maximal strain reached $s_{\max }$, the damage process is activated and the critical hourglass strain $s_{\text {max }}^{\text {hg }}$ is set as the maximal hourglass strain $s_{i j}^{h g}$ for all bonds in the computational model. In the sequential calculation, when the hourglass strain of a bond is larger than $s_{\text {max }}^{h g}$, the damage on that bond occurs, which is mathematically described as

$d_{i j}=\left\{\begin{array}{l}0 \text { if } s_{i j}^{h g}(t)>s_{\text {max }}^{h g}, t \in[0, T] \\ 1 \text { otherwise }\end{array}\right.$

where $d_{i j}$ denotes the damage status between particle $i$ and particle $j$.

The damage of a particle is calculated as

$d_{i}=\frac{\int_{\mathcal{S}_{i}} d_{i j} \mathrm{~d} V_{j}}{\int_{\mathcal{S}_{i}} \mathrm{~d} V_{j}}$.

Every time one particle is removed from the neighbour list, the nonlocal gradient for the central particle should be recalculated based on the remaining "healthy" neighbour. We will apply this rule to model fractures in 2D and 3D linear elastic material.

\section{Numerical implementation}

We have applied NOM to derive the nonlocal strong forms for the traditional continuum model in Sect. 3. Two representative nonlocal theories, the dual-horizon peridynamics by Eq. 44 for fracture modeling and the nonlocal thin plate by Eq. 60, are selected for numerical test. For the DH-PD, the focus is on the test of instability criterion for quasi-static fracture modeling by explicit time integration algorithm. The nonlocal thin plate is compared with the finite element method. The nonlocal derivatives can be viewed as a generalization of the local derivatives, and the nonlocal derivatives recover the local derivatives when the size of the support degenerates to zero. The range of nonlocality depends on the choice of the weighting functions and the size of the supports. One obstacle of the nonlocal models is the verification since the exact solutions of the nonlocal model is rare. For simplicity of verification, we aim at solving the local problems with nonlocal forms where the nonlocal effect is reduced by selecting certain weighting functions.

The primary step in the implementation is the calculation of internal force based on the governing equations. In the first step, the computational domain is discretized into particles.

$\Omega=\sum_{i=1}^{N} \Delta V_{i}$

where $N$ is the number of particles in the domain. Then the support of each particle is represented by a list of particle indices,

$\mathcal{S}_{i}=\left\{j_{1}, j_{2}, \ldots, j_{n_{i}}\right\}$

where $j$ is the global index of the particle and $n_{i}$ is the number of particles in $\mathcal{S}_{i}$.

The gradient $\boldsymbol{g}_{i j}$ and Hessian $\boldsymbol{h}_{i j}$ for two particles $i, j$ can be assembled by collecting terms in $\boldsymbol{K}_{i} \cdot \boldsymbol{p}_{i j}$ according to Eqs. 21 or 23, where

$\boldsymbol{K}_{i}=\left(\sum_{\mathcal{S}_{i}} \omega\left(\boldsymbol{r}_{i j}\right) \boldsymbol{p}_{i j} \otimes \boldsymbol{p}_{i j}^{T} \Delta V_{j}\right)^{-1}$

with weight function $\omega\left(\boldsymbol{r}_{i j}\right)=1 /\left|\boldsymbol{r}_{i j}\right|^{2}$.

The nonlocal differential derivatives at point $i$ can be calculated as

$\tilde{\partial} u_{i}=\sum_{j \in \mathcal{S}_{i}} \omega\left(\boldsymbol{r}_{i j}\right) \boldsymbol{K}_{i} \cdot \boldsymbol{p}_{i j} u_{i j} \Delta V_{j}$

The nonlocal operators in $\tilde{\partial} u_{i}$ can be used to define the strain tensor, stress tensor, bending moment and others.

In discrete form, Eqs. 44 and 58 become

$$
\begin{aligned}
\sum_{\mathcal{H}_{i}} \omega\left(\boldsymbol{r}_{i j}\right) \boldsymbol{P}_{i} \cdot \boldsymbol{g}_{i j} \Delta V_{j} \Delta V_{i} \\
\quad-\sum_{\mathcal{H}_{i}^{\prime}} \omega\left(\boldsymbol{r}_{j i}\right) \boldsymbol{P}_{j} \cdot \boldsymbol{g}_{j i} \Delta V_{j} \Delta V_{i}+\boldsymbol{b}_{i} \Delta V_{i}=\rho \Delta V_{i} \ddot{\boldsymbol{u}}_{i}
\end{aligned}
$$




$$
\begin{aligned}
\sum_{\mathcal{S}_{i}} \omega\left(\boldsymbol{r}_{i j}\right) \boldsymbol{M}_{i}: \boldsymbol{h}_{i j} \Delta V_{j} \Delta V_{i} \\
\quad-\sum_{\mathcal{S}_{i}^{\prime}} \omega\left(\boldsymbol{r}_{i j}\right) \boldsymbol{M}_{j}: \boldsymbol{h}_{j i} \Delta V_{j} \Delta V_{i}+q_{i} \Delta V_{i}=t \rho \Delta V_{i} \ddot{w}_{i}
\end{aligned}
$$

In Eqs. 93 and 94, the volume of particle $i$ is multiplied on both sides of the equations. It is not required to calculate the internal forces from the dual-support. Let $\boldsymbol{f}_{i}=\mathbf{0}, 1 \leq i \leq N$ denote the initial internal force on particle $i$. For each particle, one only needs to focus on the support, calculating the forces and adding the force to the particle internal force

$$
\begin{aligned}
\sum_{j \in \mathcal{S}_{i}} \omega\left(\boldsymbol{r}_{i j}\right) \boldsymbol{P}_{i} \cdot \boldsymbol{g}_{i j} \Delta V_{j} \Delta V_{i} & \rightarrow \boldsymbol{f}_{i} \\
-\omega\left(\boldsymbol{r}_{i j_{1}}\right) \boldsymbol{P}_{i} \cdot \boldsymbol{g}_{i j_{1}} \Delta V_{j_{1}} \Delta V_{i} & \rightarrow \boldsymbol{f}_{j_{1}} \\
-\omega\left(\boldsymbol{r}_{i j_{2}}\right) \boldsymbol{P}_{i} \cdot \boldsymbol{g}_{i j_{2}} \Delta V_{j_{2}} \Delta V_{i} & \rightarrow \boldsymbol{f}_{j_{2}} \\
\cdots & \\
-\omega\left(\boldsymbol{r}_{i j_{n_{i}}}\right) \boldsymbol{P}_{i} \cdot \boldsymbol{g}_{i j_{n_{i}}} \Delta V_{j_{n_{i}}} \Delta V_{i} & \rightarrow \boldsymbol{f}_{j_{n_{i}}}
\end{aligned}
$$

where $a \rightarrow b$ denotes the addition of $a$ to $b$. The process of adding force $-\omega\left(\boldsymbol{r}_{i j}\right) \boldsymbol{P}_{i} \cdot \boldsymbol{g}_{i j} \Delta V_{j} \Delta V_{i}$ to $\boldsymbol{f}_{j}$ is equivalent to accumulating the internal forces from particle $j$ 's dual-support.

For the calculating of internal force of thin plate, the same applies

$$
\begin{gathered}
\sum_{j \in \mathcal{S}_{i}} \omega\left(\boldsymbol{r}_{i j}\right) \boldsymbol{M}_{i}: \boldsymbol{h}_{i j} \Delta V_{j} \Delta V_{i} \rightarrow \boldsymbol{f}_{i} \\
-\omega\left(\boldsymbol{r}_{i j_{1}}\right) \boldsymbol{M}_{i}: \boldsymbol{h}_{i j_{1}} \Delta V_{j_{1}} \Delta V_{i} \rightarrow \boldsymbol{f}_{j_{1}} \\
-\omega\left(\boldsymbol{r}_{i j_{2}}\right) \boldsymbol{M}_{i}: \boldsymbol{h}_{i j_{2}} \Delta V_{j_{2}} \Delta V_{i} \rightarrow \boldsymbol{f}_{j_{2}} \\
\cdots \\
-\omega\left(\boldsymbol{r}_{i j_{n_{i}}}\right) \boldsymbol{M}_{i}: \boldsymbol{h}_{i j_{n_{i}}} \Delta V_{j_{n_{i}}} \Delta V_{i} \rightarrow \boldsymbol{f}_{j_{n_{i}}}
\end{gathered}
$$

To maintain the stability of the nonlocal operator, the discrete form of Eq. 39 is

$$
\begin{aligned}
\sum_{\mathcal{S}_{i}^{\prime}} \omega(\boldsymbol{r}) \frac{p^{h g}}{m_{j}}\left(\boldsymbol{u}_{j i}-\boldsymbol{p}_{i}^{T} \tilde{\partial} \boldsymbol{u}_{j}\right) \Delta V_{j} \Delta V_{i} \\
-\sum_{\mathcal{S}_{i}} \omega(\boldsymbol{r}) \frac{p^{h g}}{m_{i}}\left(\boldsymbol{u}_{i j}-\boldsymbol{p}_{j}^{T} \tilde{\partial} \boldsymbol{u}_{i}\right) \Delta V_{j} \Delta V_{i} .
\end{aligned}
$$

For particle $i$ with support $\mathcal{S}_{i}$, the hourglass force is calculated as follows

$$
\begin{aligned}
& \sum_{j \in \mathcal{S}_{i}} \omega\left(\boldsymbol{r}_{i j}\right) \frac{p^{h g}}{m_{i}}\left(\boldsymbol{u}_{i j}-\boldsymbol{p}_{j}^{T} \tilde{\partial} \boldsymbol{u}_{i}\right) \Delta V_{j} \Delta V_{i} \rightarrow \boldsymbol{f}_{i} \\
&-\omega\left(\boldsymbol{r}_{i j_{1}}\right) \frac{p^{h g}}{m_{i}}\left(\boldsymbol{u}_{i j_{1}}-\boldsymbol{p}_{j_{1}}^{T} \tilde{\partial} \boldsymbol{u}_{i}\right) \Delta V_{j_{1}} \Delta V_{i} \rightarrow \boldsymbol{f}_{j_{1}} \\
&-\omega\left(\boldsymbol{r}_{i j_{2}}\right) \frac{p^{h g}}{m_{i}}\left(\boldsymbol{u}_{i j_{2}}-\boldsymbol{p}_{j_{2}}^{T} \tilde{\partial} \boldsymbol{u}_{i}\right) \Delta V_{j_{2}} \Delta V_{i} \rightarrow \boldsymbol{f}_{j_{2}} \\
& \ldots \\
&-\omega\left(\boldsymbol{r}_{i j_{n_{i}}}\right) \frac{p^{h g}}{m_{i}}\left(\boldsymbol{u}_{i j_{n_{i}}}-\boldsymbol{p}_{j_{n_{i}}}^{T} \tilde{\partial} \boldsymbol{u}_{i}\right) \Delta V_{j_{n_{i}}} \Delta V_{i} \rightarrow \boldsymbol{f}_{j_{n_{i}}}
\end{aligned}
$$

When the internal force is attained and the contribution of the external force boundary condition or body force is accumulated, the basic Verlet algorithm [78] outlined as follows is used to update the displacement

$\boldsymbol{u}_{i}(t+\Delta t)=\boldsymbol{u}_{i}(t)+\boldsymbol{v}_{i}(t) \Delta t+\frac{1}{2} \boldsymbol{a}_{i}(t) \Delta t^{2}$

$v_{i}(t+\Delta t)=v_{i}(t)+\frac{1}{2}\left(a_{i}(t)+a_{i}(t+\Delta t)\right) \Delta t$

where $\boldsymbol{u}_{i}$ denotes the displacement or deflection, $\boldsymbol{v}_{i}$ the velocity and $\boldsymbol{a}_{i}=\frac{f_{i}}{m_{i}}$ the acceleration for particle $i$ with mass $m_{i}$ subject to net force $f_{i}$. For the detailed implementation and the numerical examples, the reader can find the open source code on Github https://github.com/hl-ren/Nonlocal_elast icity, and https://github.com/hl-ren/Nonlocal_thin_plate.

\section{Numerical examples}

\subsection{Accuracy of nonlocal Hessian operator}

We first test the accuracy of the nonlocal Hessian operator. Thus, consider the analytical derivatives of the field

$$
\begin{aligned}
w(x, y)= & e^{x y}(\sin 3(x-y)-\cos 2(x+y)), \\
& \text { with } x \in[-1,1], y \in[-1,1] .
\end{aligned}
$$

The domain $[-1,1]^{2}$ is discretized with different numbers of particles, $N \in\left\{20^{2}, 40^{2}, 60^{2}, 80^{2}, 100^{2}, 160^{2}, 180^{2}, 200^{2}\right\}$. The number of neighbours in support is selected as $n=14$. The $L_{2}$ norm of the nonlocal Hessian operator is calculated as

$L_{2}\left(\nabla^{2} w\right)=\sqrt{\frac{\sum_{i=1}^{N}\left(\nabla^{2} w_{i}-\tilde{\nabla}^{2} w_{i}\right):\left(\nabla^{2} w_{i}-\tilde{\nabla}^{2} w_{i}\right) \Delta V_{i}}{\sum_{i=1}^{N}\left(\nabla^{2} w_{i}\right):\left(\nabla^{2} w_{i}\right) \Delta V_{i}}}$

For different discretizations, the $L_{2}$ norm is plotted in Fig. 2a with a convergence rate of 0.835 . We also tested the influence of the support size. For fixed discretization $N=180^{2}$, 


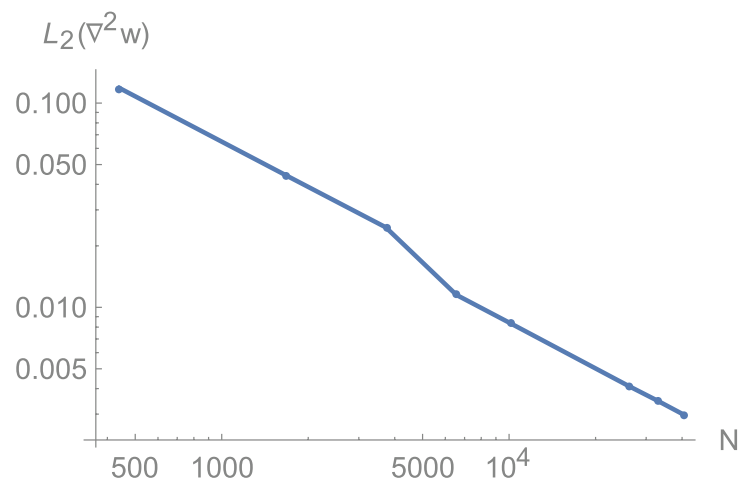

(a)

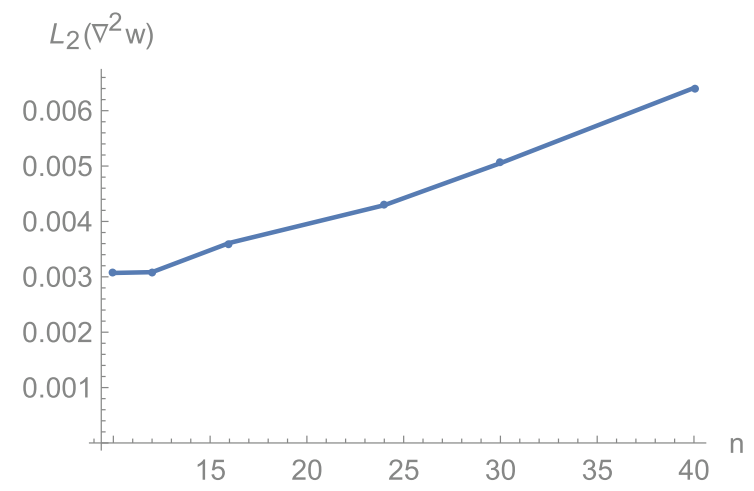

(b)

Fig. $2 L_{2}$ norm of the nonlocal Hessian operator a for $N$, the number of particles with $n=14$ and $\mathbf{b}$ for $n$, the number of particles in support with $N=180^{2}$

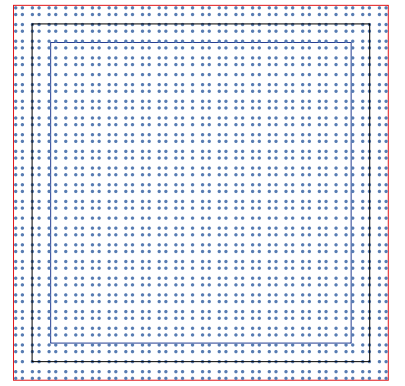

Fig. 3 The implementation of clamped boundary condition. The particles in black rectangle represent the physical model and particles outside of the blue rectangle are applied with penalty $p^{h g}=400 E$

the nonlocal effect increases with the number of neighbours in the support, as shown in Fig. $2 b$.

\subsection{Square thin plate subject to pressure}

The dimensions of the plate are $0.5 \times 0.5 \mathrm{~m}^{2}$ with a thickness of $0.01 \mathrm{~m}$. The material parameters are elastic modulus $E=210 \mathrm{GPa}$, Poisson ratio $v=0.3$. The plate is applied with a static pressure load of $p=10^{3} \mathrm{~Pa}$. Two different boundary conditions are taken into account: (a) four sides are all simply supported and (b) four sides are all clamped. The case of clamped boundary constrains the rotation as well as the deflection. The reference result is calculated by $64 \times 64$ S4R elements in ABAQUS without considering the geometrical nonlinearity. For the simply supported boundary conditions, the particles on the boundaries of the plate are fixed. The enforcement of clamped boundary conditions requires some special treatment. As shown in Fig. 3, the actual physical model of the plate is denoted by the black rectangular particles and a fictitious domain of two layers of particles outside the physical domain is generated where the particle's deflections are set to zero. the particles outside of the blue rectangle are applied with penalty $p^{h g}=400 E$ while the particles inside the blue rectangle with penalty $p^{h g}=0$. The deflection for a simply supported plate at different times are plotted in Fig. 4. The deflections for a clamped plate at different times are depicted in Fig. 5. The deflection of the central point of the plate is monitored and compared with the result by ABAQUS, as shown in Fig. 6a, b), where good agreement with FEM model is observed.

For the simply supported plate, the deflection of the central point for four different weight functions is shown in Fig. 7. It can be seen that the weight functions barely influence the results.

\subsection{Single-edge notched tension test}

In this example, we tested the nonlocal elasticity by Eq. 43 for single-edge notched tension in 2D under plane stress condition. For the case of linear elasticity, the first PiolaKirchhoff stress is the same as Cauchy stress. The geometry setup is given in Fig. 8. The bottom is fixed while the top of the plate is applied with velocity boundary condition $v=1$ $\mathrm{m} / \mathrm{s}$, which can achieve the quasi-static condition. The material parameters are $E=210 \mathrm{GPa}, v=0.3$ and critical strain is set as $s_{\max }=0.02$. The plate is discretized into $100 \times 100$ particles. Each particle's support consists of 33 nearest neighbours. The initial crack is created by modifying the neighbour list when searching the nearest neighbours. The support for each particle is constructed by finding the k-nearest neighbours and the size of the support is determined by the farthest particle in the support. Obviously, the size of the support can be different from each other. The fixed number of neighbours in support results in particles near the 


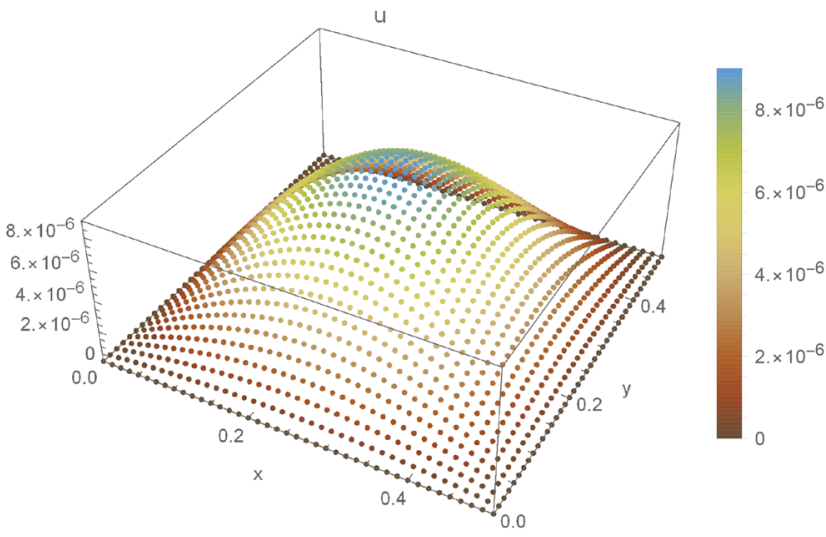

(a)

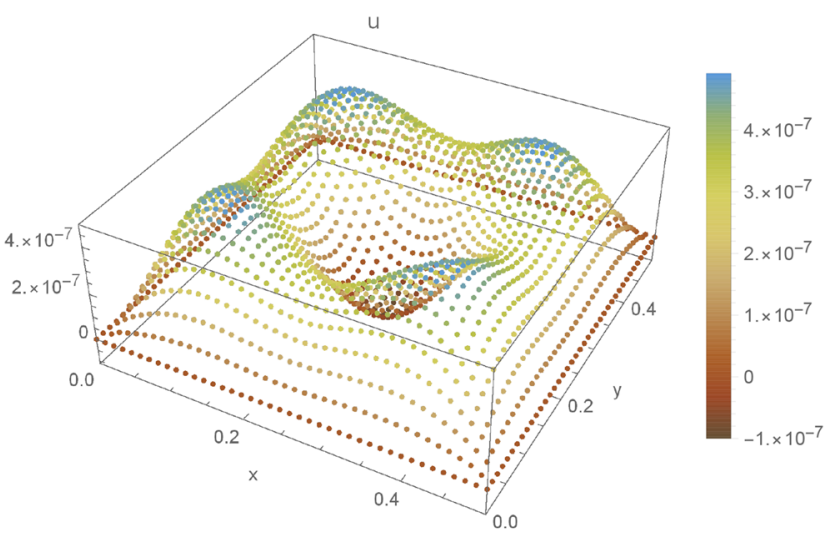

(c)

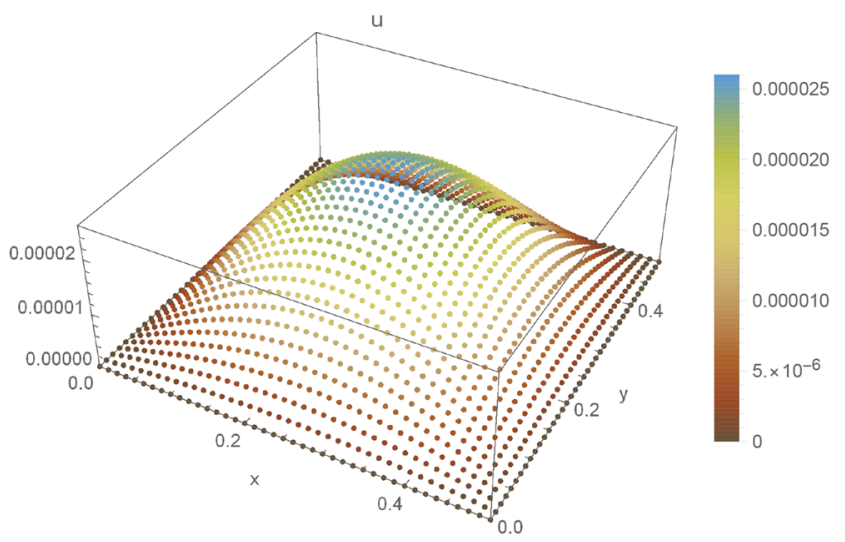

(b)

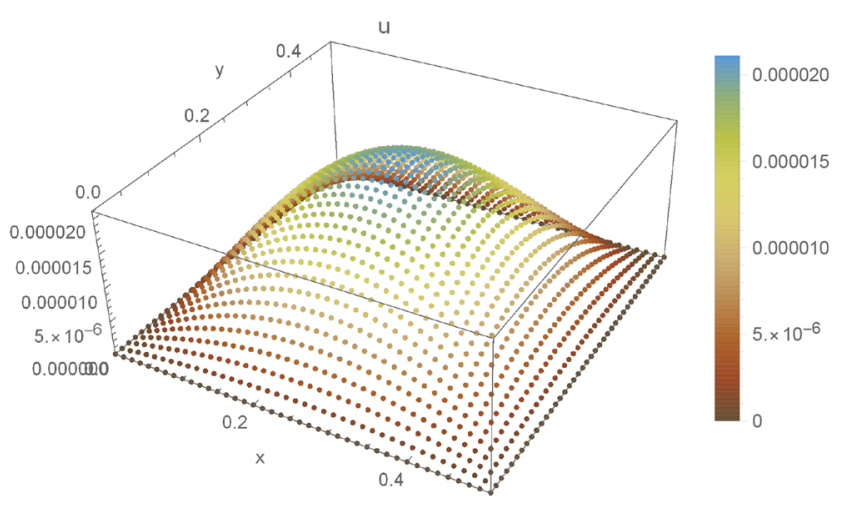

(d)

Fig. 4 Deflection of simply supported plate at $\mathbf{a} t=0.97 \mathrm{~ms}, \mathbf{b} t=2.9 \mathrm{~ms}, \mathbf{c} t=4.87 \mathrm{~ms}$ and $\mathbf{d} t=6.77 \mathrm{~ms}$

boundary with relatively large support sizes and particles at the centre of the plate with small support sizes. A duration of $T=6.5 \times 10^{-6}$ seconds is integrated by approximately 4500 steps at a time increment of $\Delta t=1.5418 \times 10^{-9} \mathrm{~s}$. Fixed velocity and displacement boundary conditions are applied to one layer of particles.

Figure $9 \mathrm{a}$ is the displacement field $u_{y}$ at full damage, where the interaction of internal force between the two half planes is cut and rigid body displacement dominates. Figure $9 \mathrm{~b}$ is the distribution of hourglass energy. We can observe that the hourglass energy is concentrated on the crack surface and crack tip. Figure 9c, d are the snapshots of damage field, which confirms that the instability criterion in Sect. 4 is stable for fracture modelling.

Although the plate is solved by an explicit dynamic method, the kinetic energy is much lower than the strain energy as shown by Fig. 10a. The dynamic load curve agrees well with that by the finite element method in Ref [77], as shown by Fig. 10b. One possible reason for the difference in reaction force increment is due to explicit algorithm and nonlocal effect of the current formulation.

\subsection{Out-of-plane shear fracture in 3D}

For brittle fracture, the basic modes of fracture are tensile fracture, in-plane shear fracture and out-of-plane shear fracture. In this section, we apply the instability damage criterion to the out-of-plane shear fracture, as shown in Fig. 11. The dimensions of the specimen are $5 \times 2.5 \times 1 \mathrm{~mm}^{3}$, as shown in Fig. 12. The size of the initial crack surface is $2.5 \times 1 \mathrm{~mm}^{2}$. The velocity boundary conditions $u_{z}=1 \mathrm{~m} / \mathrm{s}$ are applied. The model is discretized into 86,961 particles with particle size $\Delta x=0.05 \mathrm{~mm}$. Each particle has 102 neighbours in its support. Material parameters include elastic modulus $E=210 \times 10^{9} \mathrm{~Pa}$ and Poisson's ratio $v=0.3$ and density $\rho=7800 \mathrm{~kg} / \mathrm{m}^{3}$. The time step is selected as $\Delta t=7.7 \times 10^{-9} \mathrm{~s}$. A total of 3000 steps are calculated. The 


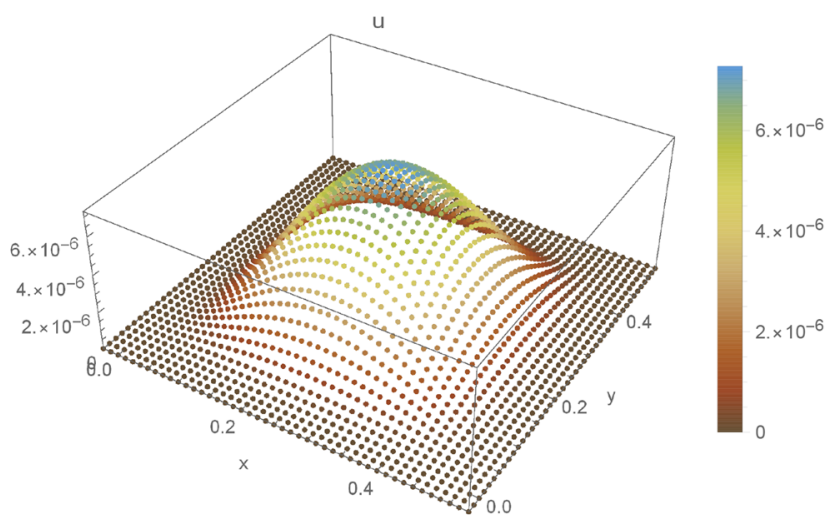

(a)

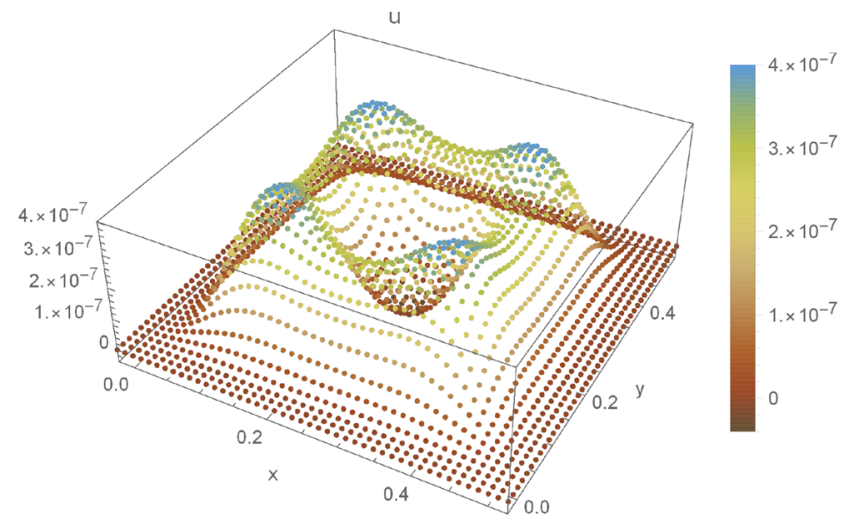

(c)

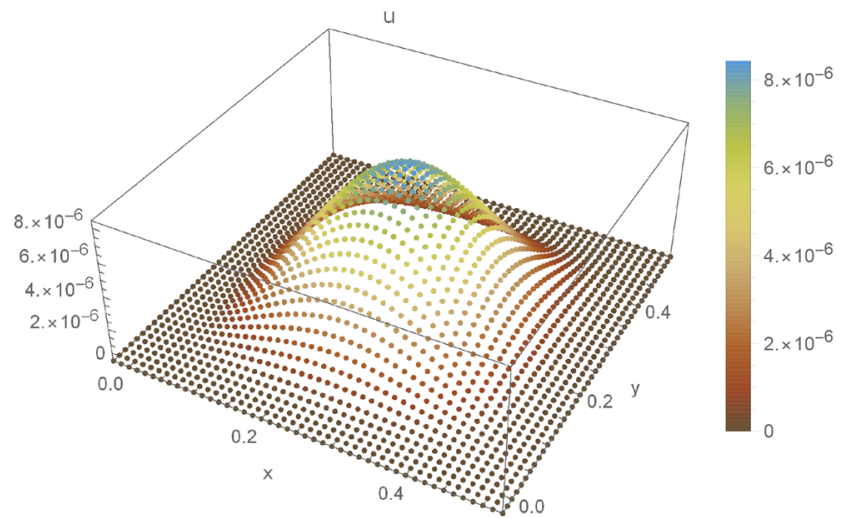

(b)

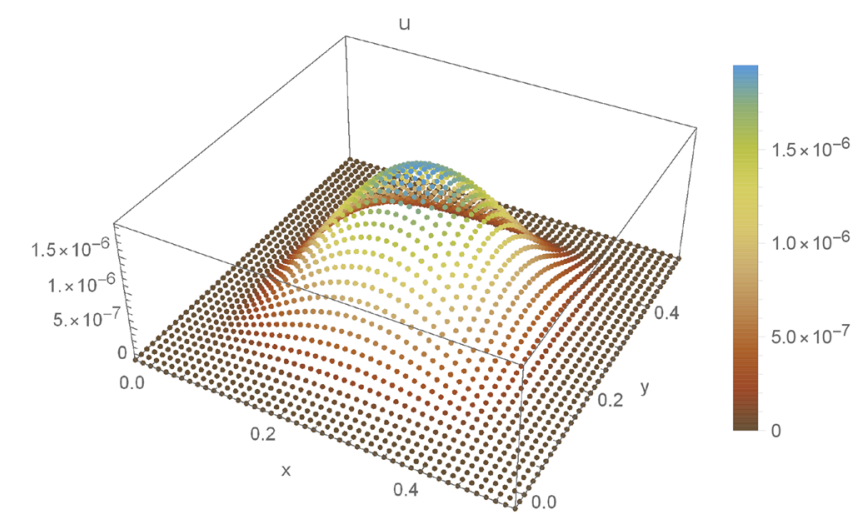

(d)

Fig. 5 Deflection of clamped plate at a $t=0.966 \mathrm{~ms}, \mathbf{b} t=1.44 \mathrm{~ms}, \mathbf{c} t=2.42 \mathrm{~ms}$ and $\mathbf{d} t=2.90 \mathrm{~ms}$

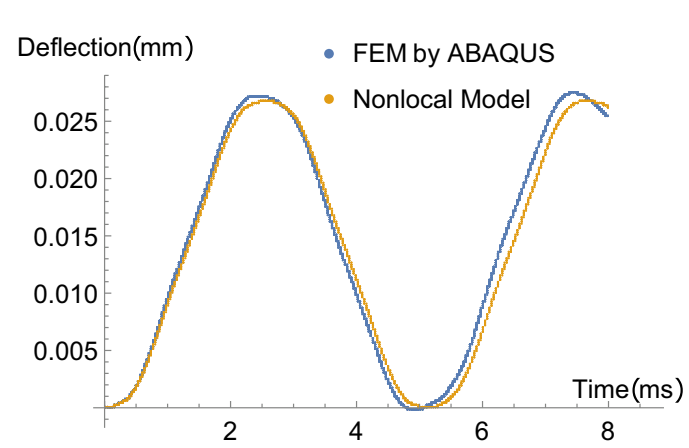

(a)

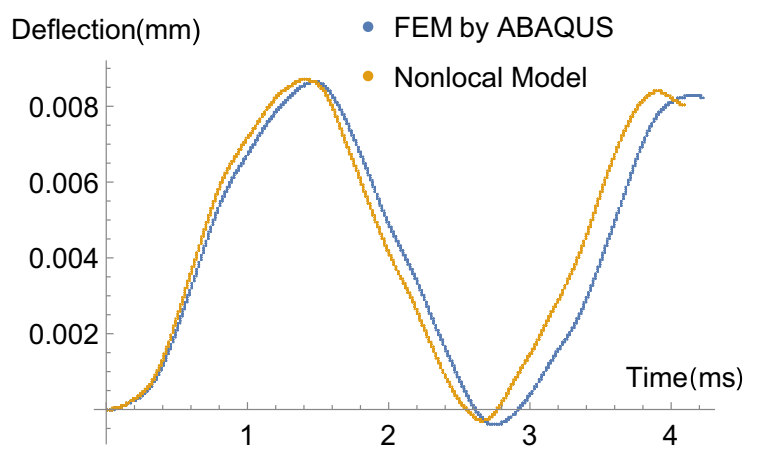

(b)

Fig. 6 Deflection of central point for a simply support plate and $\mathbf{b}$ clamped plate 


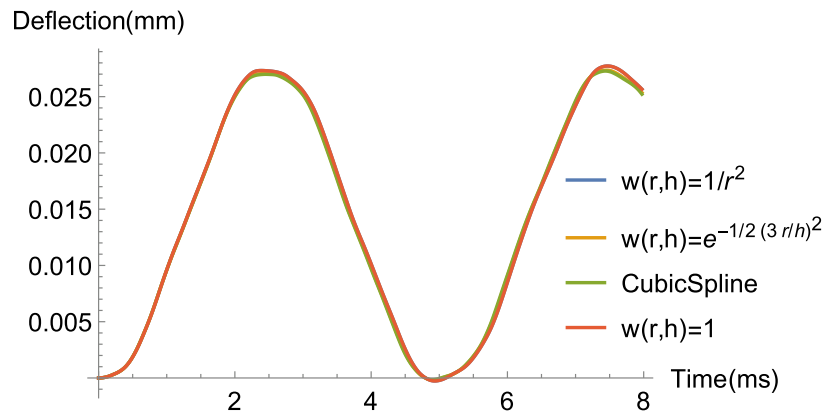

Fig. 7 Deflection of central point for simply support plate under 4 weight functions

Fig. 8 Setup of the plate

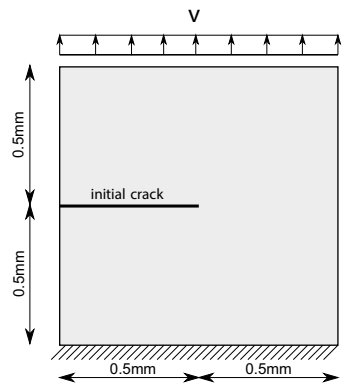

crack surface starts to propagate at step 1550. The crack surface at different steps are depicted in Fig. 13.

\section{Conclusion}

In this paper, we employ the recently proposed NOM to derive the nonlocal strong forms for various physical models, including elasticity, thin plate, gradient elasticity, electro-magneto-elastic coupled model and phase-field fracture model. These models require a second-order partial derivative at most and we make use of the second-order NOM scheme, which contains the nonlocal gradient and nonlocal Hessian operator. Considering the fact that most physical models are compatible with the variational principle/ weighted residual method, we start from the energy form/ weak form of the problem, by inserting the nonlocal expression of the gradient/Hessian operator into the weak form, based on the dual property of the dual-support in NOM, the nonlocal strong form is obtained with ease. Such a process can be extended to many other physical problems in other fields. The derived strong forms are variationally consistent and allow elegant description for inhomogeneous nonlocality in both theoretical derivation and numerical implementation.

We also propose an instability criterion in nonlocal elasticity or dual-horizon state-based peridynamics for the fracture modeling. The criterion is formulated as the functional of nonlocal gradient in support, which minimizes the zero-energy deformation that cannot be described by the nonlocal gradient. Such an operator functional approaches zero for continuous fields but has comparable value to the strain energy density for the deformation around the crack tip. During the fracture modeling by removing particles from the neighbor list, it is safer to delete the particle with larger zero-energy deformation. The numerical examples for 2D/3D fracture modeling confirm the feasibility and robustness of this criterion. The instability criterion is applicable for anisotropic elastic material and hyperelastic materials.

\section{Appendix A: A simple example to illustrate dual-support}

In order to facilitate the comprehension of dual-support, let us consider 4 particles in Fig. 14, each with particle volume $\Delta V_{i}, i=\{1,2,3,4\}$ and $\Omega=\sum_{i=1}^{4} \Delta V_{i}$. Obviously, the support and dual-support can be listed as follows.

$\mathcal{S}_{1}=\{2,3,4\}, \mathcal{S}_{1}^{\prime}=\{3,4\}$

$\mathcal{S}_{2}=\{3\}, \mathcal{S}_{2}^{\prime}=\{1,3\}$

$\mathcal{S}_{3}=\{1,2\}, \mathcal{S}_{3}^{\prime}=\{1,2,4\}$

$\mathcal{S}_{4}=\{1,3\}, \mathcal{S}_{4}^{\prime}=\{1\}$

Here we neglect whether the shape tensor is invertible or not.

The most common formula in the derivation based on NOM and variational principle is the double integrations in support and whole domain. Consider the double integrations

$$
\begin{aligned}
& \int_{\Omega} \int_{\mathcal{S}_{i}} f_{i j}\left(u_{j}-u_{i}\right) \mathrm{d} V_{j} \mathrm{~d} V_{i} \\
& \approx \sum_{i=1}^{4}\left(\sum_{j \in \mathcal{S}_{i}} f_{i j}\left(u_{j}-u_{i}\right) \Delta V_{j}\right) \Delta V_{i} \\
& =\sum_{i=1}^{4}\left(\sum_{j \in \mathcal{S}_{i}} f_{i j} u_{j} \Delta V_{j}\right) \Delta V_{i}-\sum_{i=1}^{4}\left(\sum_{j \in \mathcal{S}_{i}} f_{i j} u_{i} \Delta V_{j}\right) \Delta V_{i}
\end{aligned}
$$

Expand the double summations 


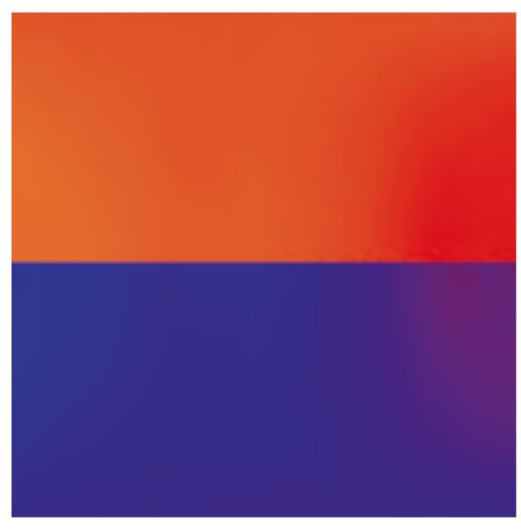

(a)

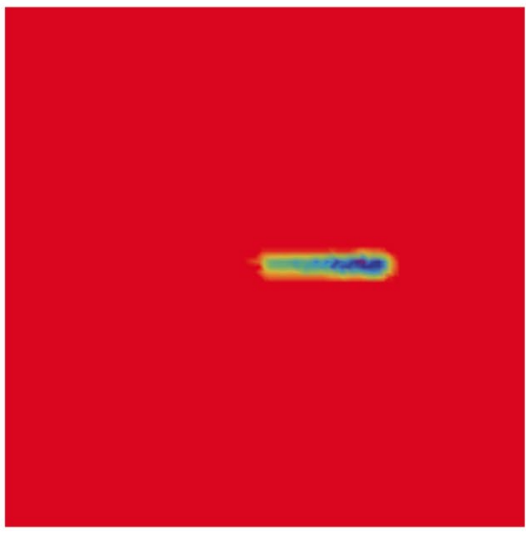

(c)

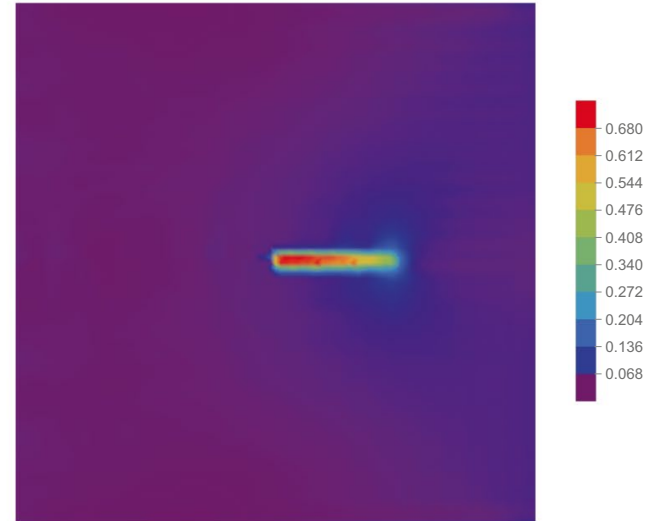

(b)

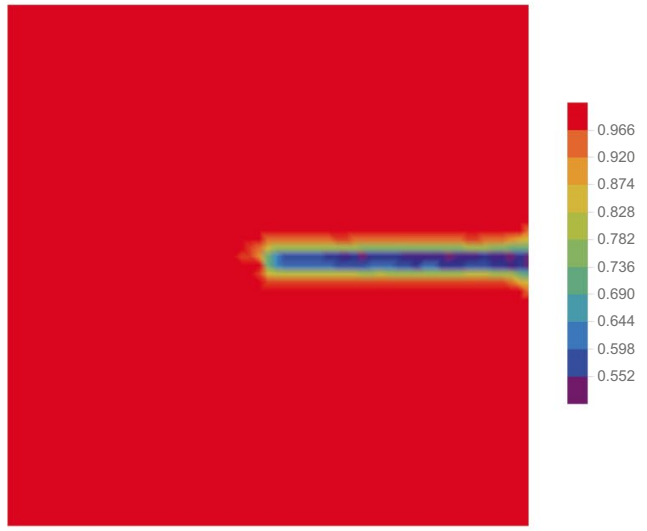

(d)

Fig. 9 a Displacement $u_{y}$ at full damage, b operator energy at $u_{y}=5.5 \times 10^{-3} \mathrm{~mm}$, c damage field at $u_{y}=5.5 \times 10^{-3} \mathrm{~mm}$ and $\mathbf{d}$ damage field at $u_{y}=6.2 \times 10^{-3} \mathrm{~mm}$

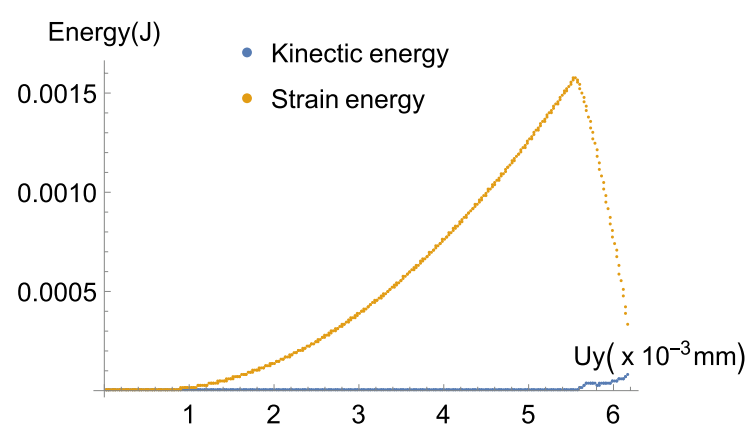

(a)

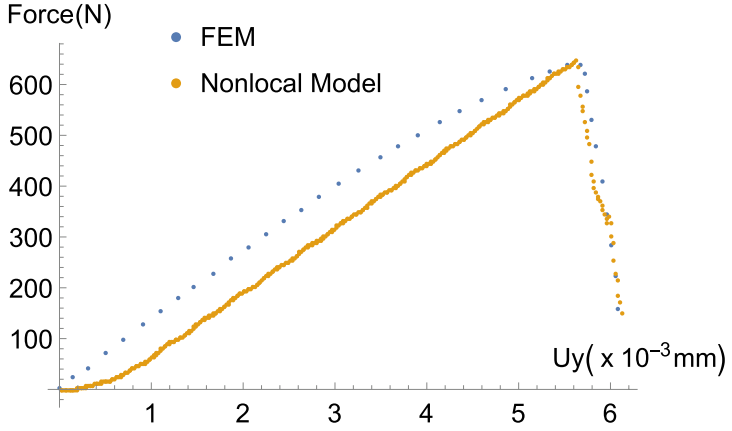

(b)

Fig. 10 a Energy curve on displacement; b load curve on displacement 


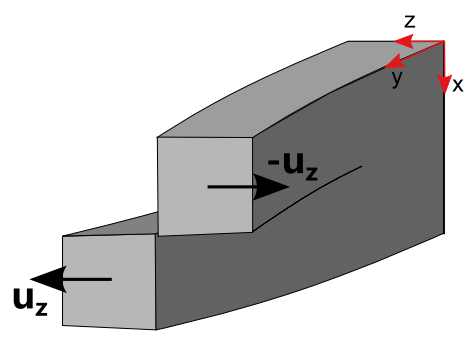

Fig. 11 Illustration of out-of-plane shear fracture

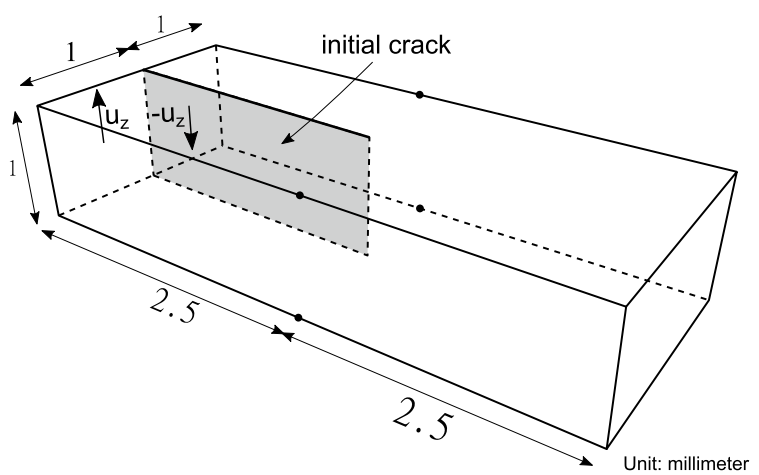

Fig. 12 Setup of the specimen

Fig. 13 Crack surfaces at a step 1550, b step 2050, c step 2950 and d step 3000

$$
\begin{aligned}
\sum_{i=1}^{4} & \left(\sum_{j \in \mathcal{S}_{i}} f_{i j} u_{j} \Delta V_{j}\right) \Delta V_{i} \\
= & \left(f_{12} \Delta V_{2} \Delta V_{1} u_{2}+f_{13} \Delta V_{3} \Delta V_{1} u_{3}+f_{14} \Delta V_{4} \Delta V_{1} u_{4}\right) \\
& +\left(f_{23} \Delta V_{2} \Delta V_{3} u_{3}\right) \\
& +\left(f_{31} \Delta V_{1} \Delta V_{3} u_{1}+f_{32} \Delta V_{2} \Delta V_{3} u_{2}\right) \\
& +\left(f_{41} \Delta V_{1} \Delta V_{4} u_{1}+f_{43} \Delta V_{3} \Delta V_{4} u_{3}\right) \\
= & \left(f_{31} \Delta V_{3}+f_{41} \Delta V_{4}\right) u_{1} \Delta V_{1} \\
& +\left(f_{12} \Delta V_{1}+f_{32} \Delta V_{3}\right) u_{2} \Delta V_{2} \\
& +\left(f_{13} \Delta V_{1}+f_{23} \Delta V_{2}+f_{43} \Delta V_{4}\right) u_{3} \Delta V_{3} \\
& +\left(f_{14} \Delta V_{1}\right) u_{4} \Delta V_{4} \\
= & \sum_{j \in S_{1}^{\prime}} f_{j 1} \Delta V_{j} \Delta V_{1} u_{1}+\sum_{j \in S_{2}^{\prime}} f_{j 2} \Delta V_{j} \Delta V_{2} u_{2} \\
& +\sum_{j \in S_{3}^{\prime}} f_{j 3} \Delta V_{j} \Delta V_{3} u_{3}+\sum_{j \in S_{4}^{\prime}} f_{j 4} \Delta V_{j} \Delta V_{4} u_{4} \\
= & \sum_{i=1}^{4}\left(\sum_{j \in \mathcal{S}_{i}^{\prime}} f_{j i} \Delta V_{j}\right) u_{i} \Delta V_{i} . \\
&
\end{aligned}
$$

(a)
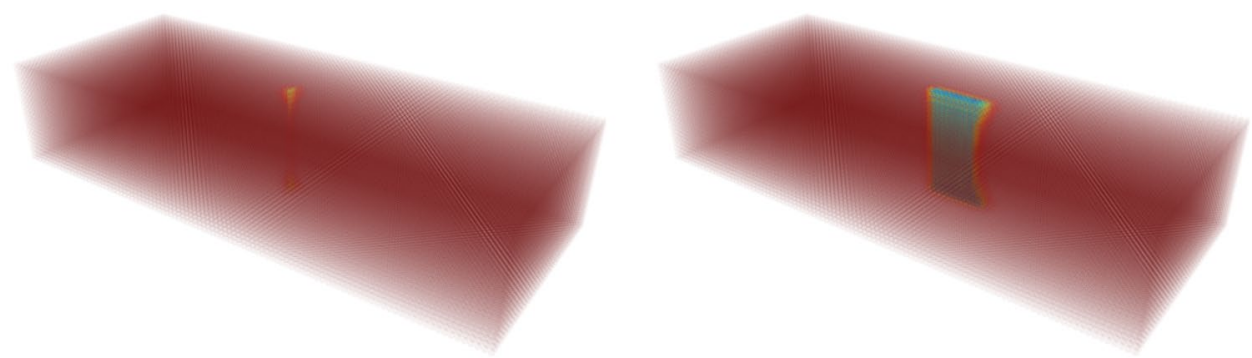

(b)

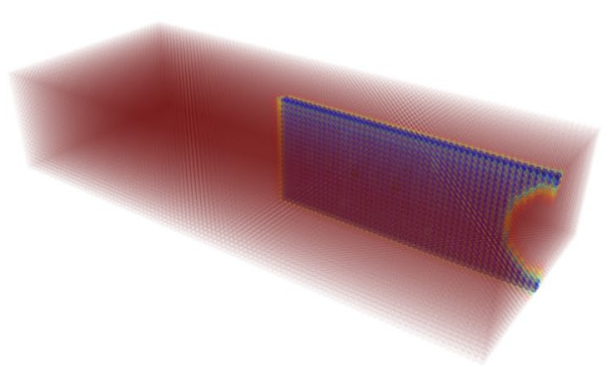

(c)

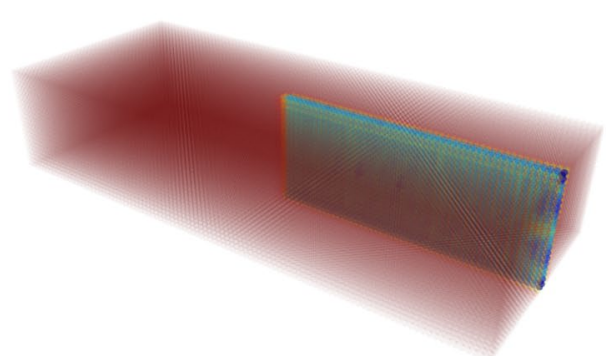

(d) 


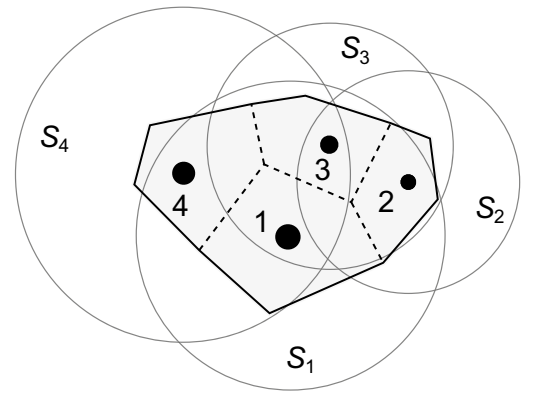

Fig. 14 Particles 1-4 and their supports $\mathcal{S}_{i}, i=\{1,2,3,4\}$

Therefore

$$
\begin{aligned}
\sum_{i=1}^{4} & \left(\sum_{j \in \mathcal{S}_{i}} f_{i j} u_{j} \Delta V_{j}\right) \Delta V_{i} \\
& -\sum_{i=1}^{4}\left(\sum_{j \in \mathcal{S}_{i}} f_{i j} u_{i} \Delta V_{j}\right) \Delta V_{i} \\
= & \sum_{i=1}^{4}\left(\sum_{j \in \mathcal{S}_{i}^{\prime}} f_{j i} \Delta V_{j}\right) u_{i} \Delta V_{i} \\
& -\sum_{i=1}^{4}\left(\sum_{j \in \mathcal{S}_{i}} f_{i j} u_{i} \Delta V_{j}\right) \Delta V_{i} \\
= & \sum_{i=1}^{4}\left(\sum_{j \in \mathcal{S}_{i}^{\prime}} f_{j i} \Delta V_{j}\right. \\
& \left.-\sum_{j \in \mathcal{S}_{i}} f_{i j} \Delta V_{j}\right) u_{i} \Delta V_{i} \\
\approx & \int_{\Omega}\left(\int_{\mathcal{S}_{i}^{\prime}} f_{j i} \mathrm{~d} V_{j}-\int_{\mathcal{S}_{i}} f_{i j} \mathrm{~d} V_{j}\right) u_{i} \mathrm{~d} V_{i} .
\end{aligned}
$$

At last, we obtain

$$
\begin{gathered}
\int_{\Omega} \int_{\mathcal{S}_{i}} f_{i j}\left(u_{j}-u_{i}\right) \mathrm{d} V_{j} \mathrm{~d} V_{i}=\int_{\Omega} \\
\left(\int_{\mathcal{S}_{i}^{\prime}} f_{j i} \mathrm{~d} V_{j}-\int_{\mathcal{S}_{i}} f_{i j} \mathrm{~d} V_{j}\right) u_{i} \mathrm{~d} V_{i} .
\end{gathered}
$$

Above equation is widely used in the derivation of nonlocal strong form from weak form. Such expression is valid in the continuum form as well as in discrete form.

Funding Open Access funding enabled and organized by Projekt DEAL.

Open Access This article is licensed under a Creative Commons Attribution 4.0 International License, which permits use, sharing, adaptation, distribution and reproduction in any medium or format, as long as you give appropriate credit to the original author(s) and the source, provide a link to the Creative Commons licence, and indicate if changes were made. The images or other third party material in this article are included in the article's Creative Commons licence, unless indicated otherwise in a credit line to the material. If material is not included in the article's Creative Commons licence and your intended use is not permitted by statutory regulation or exceeds the permitted use, you will need to obtain permission directly from the copyright holder. To view a copy of this licence, visit http://creativecommons.org/licenses/by/4.0/.

\section{References}

1. Toupin RA (1962) Elastic materials with couple-stresses. Arch Ration Mech Anal 11(1):385-414

2. Mindlin RD (1964) Micro-structure in linear elasticity. Arch Ration Mech Anal 16:51-78

3. Davydov D, Javili A, Steinmann P (2013) On molecular statics and surface-enhanced continuum modeling of nano-structures. Comput Mater Sci 69:510-519

4. Areias P, Lopes JC, Santos MP, Rabczuk T, Reinoso J (2019) Finite strain analysis of limestone/basaltic magma interaction and fracture: low order mixed tetrahedron and remeshing. Eur J Mech A/Solids 73:235-247

5. Sukumar N, Moës N, Moran B, Belytschko T (2000) Extended finite element method for three-dimensional crack modelling. Int J Numer Methods Eng 48(11):1549-1570

6. Nguyen VP, Wu JY (2018) Modeling dynamic fracture of solids with a phase-field regularized cohesive zone model. Comput Methods Appl Mech Eng 340:1000-1022

7. Ren HL, Zhuang XY, Anitescu C, Rabczuk T (2019) An explicit phase field method for brittle dynamic fracture. Comput Struct 217:45-56

8. Zhou SW, Zhuang XY (2020) Phase field modeling of hydraulic fracture propagation in transversely isotropic poroelastic media. Acta Geotech 15(9)

9. Rabczuk T, Belytschko T (2004) Cracking particles: a simplified meshfree method for arbitrary evolving cracks. Int J Numer Methods Eng 61(13):2316-2343

10. Zhang YM, Zhuang XY (2018) Cracking elements: a self-propagating strong discontinuity embedded approach for quasi-brittle fracture. Finite Elem Anal Des 144:84-100

11. Majidi HR, Ayatollahi MR, Torabi AR (2018) On the use of the extended finite element and incremental methods in brittle fracture assessment of key-hole notched polystyrene specimens under mixed mode i/ii loading with negative mode i contributions. Arch Appl Mech 88(4):587-612

12. Yang YT, Sun GH, Zheng H (2019) Stability analysis of soil-rockmixture slopes using the numerical manifold method. Eng Anal Bound Elem 109:153-160

13. Singh SK, Singh IV, Bhardwaj G, Mishra BK (2018) A bézier extraction based XIGA approach for three-dimensional crack simulations. Adv Eng Softw 125:55-93

14. Belytschko T, Lu YY, Gu L (1994) Element-free galerkin methods. Int J Numer Methods Eng 37(2):229-256

15. Liu WK, Jun S, Zhang YF (1995) Reproducing kernel particle methods. Int J Numer Methods Fluids 20(8-9):1081-1106

16. Huerta A, Belytschko T, Fernández-Méndez S, Rabczuk T, Zhuang XY, Arroyo M (2018) Meshfree methods. In: Encyclopedia of computational mechanics, 2nd edn, pp 1-38

17. Mindlin RD, Eshel NN (1968) On first strain-gradient theories in linear elasticity. Int J Solids Struct 4(1):109-124

18. Yang F, Chong ACM, Lam DCC, Tong P (2002) Couple stress based strain gradient theory for elasticity. Int J Solids Struct 39(10):2731-2743 
19. Polizzotto C (2012) A gradient elasticity theory for secondgrade materials and higher order inertia. Int J Solids Struct 49(15-16):2121-2137

20. Nguyen Hoang X, Nguyen Tuan N, Abdel-Wahab Magd, Bordas Stéphane PA, Nguyen-Xuan Hung, Vo Thuc P (2017) A refined quasi-3d isogeometric analysis for functionally graded microplates based on the modified couple stress theory. Comput Methods Appl Mech Eng 313:904-940

21. Eringen AC (1972) Nonlocal polar elastic continua. Int J Eng Sci 10(1):1-16

22. Eringen AC, Edelen DGB (1972) On nonlocal elasticity. Int J Eng Sci 10(3):233-248

23. Eringen AC (1983) On differential equations of nonlocal elasticity and solutions of screw dislocation and surface waves. J Appl Phys 54(9):4703-4710

24. Eringen AC (2012) Microcontinuum field theories: I. Foundations and solids. Springer Science \& Business Media, Berlin

25. Cosserat E, Cosserat F (1909) Théorie des corps déformables. A. Hermann et fils

26. Toupin RA (1964) Theories of elasticity with couple-stress. Arch Ration Mech Anal 17(2):85-112

27. Tsiatas GC (2009) A new Kirchhoff plate model based on a modified couple stress theory. Int J Solids Struct 46(13):2757-2764

28. Dell'Isola F, Andreaus U, Placidi L (2015) At the origins and in the vanguard of peridynamics, non-local and higher-gradient continuum mechanics: an underestimated and still topical contribution of Gabrio Piola. Math Mech Solids 20(8):887-928

29. Silling SA (2000) Reformulation of elasticity theory for discontinuities and long-range forces. J Mech Phys Solids 48(1):175-209

30. Silling SA, Epton M, Weckner O, Xu J, Askari E (2007) Peridynamic states and constitutive modeling. J Elast 88(2):151-184

31. Foster JT, Silling SA, Chen WN (2011) An energy based failure criterion for use with peridynamic states. Int J Multiscale Comput Eng 9(6)

32. Liu WY, Yang G, Cai Y (2018) Modeling of failure mode switching and shear band propagation using the correspondence framework of peridynamics. Comput Struct 209:150-162

33. Zhou XP, Wang YT, Xu XM (2016) Numerical simulation of initiation, propagation and coalescence of cracks using the nonordinary state-based peridynamics. Int J Fract 201(2):213-234

34. Zhou XP, Wang YT, Qian QH (2016) Numerical simulation of crack curving and branching in brittle materials under dynamic loads using the extended non-ordinary state-based peridynamics. Eur J Mech A/Solids 60:277-299

35. Ren HL, Zhuang XY, Rabczuk T (2016) A new peridynamic formulation with shear deformation for elastic solid. J Micromech Mol Phys 1(02):1650009

36. Zhu QZ, Ni T (2017) Peridynamic formulations enriched with bond rotation effects. Int J Eng Sci 121:118-129

37. Diana V, Casolo S (2019) A bond-based micropolar peridynamic model with shear deformability: elasticity, failure properties and initial yield domains. Int J Solids Struct 160:201-231

38. Silling SA, Lehoucq RB (2010) Peridynamic theory of solid mechanics. Adv Appl Mech 44:73-168

39. Gu X, Zhang Q, Madenci E, Xia XZ (2019) Possible causes of numerical oscillations in non-ordinary state-based peridynamics and a bond-associated higher-order stabilized model. Comput Methods Appl Mech Eng 357:112592

40. Yaghoobi A, Chorzepa MG (2017) Higher-order approximation to suppress the zero-energy mode in non-ordinary state-based peridynamics. Comput Struct 188:63-79

41. Silling SA (2017) Stability of peridynamic correspondence material models and their particle discretizations. Comput Methods Appl Mech Eng 322:42-57
42. Li P, Hao ZM, Zhen WQ (2018) A stabilized non-ordinary statebased peridynamic model. Comput Methods Appl Mech Eng 339:262-280

43. Chowdhury SR, Roy P, Roy D, Reddy JN (2019) A modified peridynamics correspondence principle: removal of zero-energy deformation and other implications. Comput Methods Appl Mech Eng 346:530-549

44. Cui H, Li CG, Zheng H (2020) A higher-order stress point method for non-ordinary state-based peridynamics. Eng Anal Bound Elem 117:104-118

45. Ren HL, Zhuang XY, Cai YC, Rabczuk T (2016) Dual-horizon peridynamics. Int J Numer Methods Eng 108(12):1451-1476

46. Ren HL, Zhuang XY, Rabczuk T (2017) Dual-horizon peridynamics: a stable solution to varying horizons. Comput Methods Appl Mech Eng 318:762-782

47. Taylor M, Steigmann DJ (2015) A two-dimensional peridynamic model for thin plates. Math Mech Solids 20(8):998-1010

48. Chowdhury SR, Roy P, Roy D, Reddy JN (2016) A peridynamic theory for linear elastic shells. Int J Solids Struct 84:110-132

49. Dorduncu M (2019) Stress analysis of laminated composite beams using refined zigzag theory and peridynamic differential operator. Compos Struct 218:193-203

50. Zhang Q, Li SF, Zhang AM, Peng YX, Yan JL (2021) A peridynamic reissner-mindlin shell theory. Int J Numer Methods Eng 122(1):122-147

51. Bode T, Weißenfels C, Wriggers P (2020) Peridynamic PetrovGalerkin method: a generalization of the peridynamic theory of correspondence materials. Comput Methods Appl Mech Eng $358: 112636$

52. Bode T, Weißenfels C, Wriggers P (2020) Mixed peridynamic formulations for compressible and incompressible finite deformations. Comput Mech 65(5):1365-1376

53. Roy P, Deepu SP, Pathrikar A, Roy D, Reddy JN (2017) Phase field based peridynamics damage model for delamination of composite structures. Compos Struct 180:972-993

54. Butt SN, Timothy JJ, Meschke G (2017) Wave dispersion and propagation in state-based peridynamics. Comput Mech 60(5):725-738

55. Yu HC, Li SF (2020) On energy release rates in peridynamics. J Mech Phys Solids 142:104024

56. Bie YH, Cui XY, Li ZC (2018) A coupling approach of statebased peridynamics with node-based smoothed finite element method. Comput Methods Appl Mech Eng 331:675-700

57. D'Elia M, Li XJ, Seleson P, Tian XC, Yu Y (2019) A review of local-to-nonlocal coupling methods in nonlocal diffusion and nonlocal mechanics. arXiv: 1912.06668

58. Chen HL, Chan WL (2020) Higher-order peridynamic material correspondence models for elasticity. J Elast 142(1):135-161

59. Madenci Erdogan, Barut Atila, Dorduncu Mehmet (2019) Peridynamic differential operator for numerical analysis. Springer, Berlin

60. Madenci Erdogan, Dorduncu Mehmet, Xin Gu (2019) Peridynamic least squares minimization. Comput Methods Appl Mech Eng 348:846-874

61. Madenci Erdogan, Dorduncu Mehmet, Barut Atila, Futch Michael (2017) Numerical solution of linear and nonlinear partial differential equations using the peridynamic differential operator. Numer Methods Partial Differ Equ 33(5):1726-1753

62. Gao Yan, Oterkus Selda (2019) Non-local modeling for fluid flow coupled with heat transfer by using peridynamic differential operator. Eng Anal Bound Elem 105:104-121

63. Wang Hanlin, Oterkus Erkan, Oterkus Selda (2018) Three-dimensional peridynamic model for predicting fracture evolution during the lithiation process. Energies 11(6):1461

64. Wang BQ, Oterkus S, Oterkus E (2020) Derivation of dual-horizon state-based peridynamics formulation based on Euler-Lagrange 
equation. Continuum Mech Thermodyn. https://doi.org/10.1007/ s00161-020-00915-y

65. Ren HL, Zhuang XY, Rabczuk Timon (2020) A nonlocal operator method for solving partial differential equations. Comput Methods Appl Mech Eng 358:112621

66. Rabczuk T, Ren HL, Zhuang X (2019) A nonlocal operator method for partial differential equations with application to electromagnetic waveguide problem. Comput Mater Continua 59(1):2019

67. Ren HL, Zhuang XY, Rabczuk Timon (2020) A higher order nonlocal operator method for solving partial differential equations. Comput Methods Appl Mech Eng 367:113132

68. Ren HL, Zhuang XY, Rabczuk Timon (2020) Nonlocal operator method with numerical integration for gradient solid. Comput Struct 233:106235

69. Ren HL, Zhuang XY, Trung NT, Rabczuk T (2020) Nonlocal operator method for the Cahn-Hilliard phase field model. Commun Nonlinear Sci Numer Simul 96:105687

70. Wang YT, Zhou XP, Wang Y, Shou YD (2018) A 3-D conjugated bond-pair-based peridynamic formulation for initiation and propagation of cracks in brittle solids. Int J Solids Struct 134:89-115

71. Javili A, Firooz S, McBride AT, Steinmann P (2020) The computational framework for continuum-kinematics-inspired peridynamics. Comput Mech 66(4):795-824

72. Ren Huilong, Zhuang Xiaoying, Trung Nguyen-Thoi, Rabczuk Timon (2021) A nonlocal operator method for finite deformation higher-order gradient elasticity. Comput Methods Appl Mech Eng 384:113963

73. Timoshenko SP, Woinowsky-Krieger S (1959) Theory of plates and shells. McGraw-Hill, New York

74. Liu LP (2014) An energy formulation of continuum magneto-electro-elasticity with applications. J Mech Phys Solids 63:451-480

75. Nguyen BH, Zhuang XY, Rabczuk T (2019) Nurbs-based formulation for nonlinear electro-gradient elasticity in semiconductors. Comput Methods Appl Mech Eng 346:1074-1095

76. Roy P, Roy D, Reddy JN (2019) A conformal gauge theory of solids: insights into a class of electromechanical and magnetomechanical phenomena. J Mech Phys Solids 130:35-55

77. Miehe C, Welschinger F, Hofacker M (2010) Thermodynamically consistent phase-field models of fracture: variational principles and multi-field fe implementations. Int J Numer Methods Eng 83(10):1273-1311

78. Verlet L (1967) Computer "experiments" on classical fluids. I. Thermodynamical properties of lennard-jones molecules. Phys Rev 159(1):98

Publisher's Note Springer Nature remains neutral with regard to jurisdictional claims in published maps and institutional affiliations. 